\title{
Conduits based on the combination of hyaluronic acid and silk fibroin: characterization, in vitro studies and in vivo biocompatibility
}

\author{
F. Gisbert Roca ${ }^{\mathrm{a}}$, P. Lozano Picazo ${ }^{\mathrm{c}, \mathrm{d}}$, J. Pérez-Rigueiro ${ }^{\mathrm{b}, \mathrm{c}, \mathrm{d}}$, G.V. Guinea Tortuero ${ }^{\mathrm{b}, \mathrm{c}, \mathrm{d}}$, \\ M. Monleón Pradas ${ }^{\mathrm{a}, \mathrm{b}}$ and C. Martínez-Ramos, ${ }^{\mathrm{a},}$
}

\begin{abstract}
a. Center for Biomaterials and Tissue Engineering, Universitat Politècnica de València, Cno. de Vera s/n, 46022, Valencia, Spain
b. CIBER-BBN, Biomedical Research Networking Center in Bioengineering Biomaterials and Nanomedicine, Spain

c. Centro de Tecnología Biomédica. Universidad Politécnica de Madrid. 28223 Pozuelo de Alarcón (Madrid), Spain

d. Departamento de Ciencia de Materiales. ETSI Caminos, Canales y Puertos., Universidad Politécnica de Madrid, 28040, Madrid.

*Corresponding author: Cristina Martínez Ramos. Centro de Biomateriales e Ingeniería Tisular, C.P.I. Edificio 8E, Acceso F, Nivel

1, Universidad Politécnica de Valencia, Camino de Vera s/n E-46022 Valencia, España. Tel.: +34963877000; fax: +34963877276.

E-mail: cris mr1980@hotmail.com.
\end{abstract}

\begin{abstract}
We address the production of structures intended as conduits made from natural biopolymers, capable of promoting the regeneration of axonal tracts. We combine hyaluronic acid (HA) and silk fibroin (SF) with the aim of improving mechanical and biological properties of HA. The results show that SF can be efficiently incorporated into the production process, obtaining conduits with tubular structure with a matrix of HA-SF blend. HA-SF has better mechanical properties than sole HA, which is a very soft hydrogel, facilitating manipulation. Culture of rat Schwann cells shows that cell adhesion and proliferation are higher than in pure HA, maybe due to the binding motifs contributed by the SF protein. This increased proliferation accelerates the formation of a tight cell layer, which covers the inner channel surface of the HA-SF tubes. Biocompatibility of the scaffolds was studied in immunocompetent mice. Both HA and HA-SF scaffolds were accepted by the host with no residual immune response at 8 weeks. New collagen extracellular matrix and new blood vessels were visible and they were present earlier when SF was present. The results show that incorporation of SF enhances the mechanical properties of the materials and results in promising biocompatible conduits for tubulization strategies.
\end{abstract}

Keywords: biomaterials, hyaluronic acid, silk fibroin, tissue engineering, nerve guidance conduits. 


\section{Introduction}

Injuries and diseases affecting the nervous system have very high incidence and prevalence, affecting millions of people around the world. New concepts and solutions are in demand for both the central nervous system (CNS) and the peripheral nervous system (PNS). In the human CNS axons do not regenerate spontaneously after damage or degeneration, causing life-long functional deficit as in the case of spinal cord injury, traumatic brain damage or cerebral infarction [1]. Failure of axonal regeneration is probably due, among other factors, to the formation of an impenetrable glial scar, composed mainly of astrocytes, that inhibits axonal growth and myelination [2]. This causes regenerative neurons to be blocked and unable to reach their synaptic target. In the PNS there is successful endogenous axon regeneration in adult humans for distances of few centimetres [1]. Here damage is followed by a degeneration of the distal segment of the nerve, known as Wallerian degeneration, while the proximal segment of the nerve suffers only minimal damage $[3,4]$. After the distal segment degeneration, Schwann cells and phagocytic cells such as macrophages remove myelin and axonal debris while producing cytokines that improve the axonal growth, creating a growth cone able to direct axon regrowth until reconnection with the distal target. Finally, Schwann cells myelinate the re-growing axon forming new myelin sheaths $[4,5]$. Nonetheless, in cases of larger damages the process of regeneration is not successful.

In order to improve over the limitations of current therapies tissue engineering approaches invoke the combination of cell supply, bioactive molecules and scaffolds based on biomaterials [6,7]. conduits typically consist in hollow tubes used to connect the two ends of a damaged nerve, guiding the axonal regeneration between both sides of the injury [8]. Their main goal is to protect the spontaneous regeneration process. Conduits must guide tissue regeneration from one end to the other of the lesion, avoiding the growth of fibrous tissue and retaining the neurotrophic factors secreted by the damaged nerve remnants [9]. Tubular scaffolds should have a porosity large enough to allow the exchange of oxygen and nutrients, and small enough to prevent the infiltration of inflammatory cells into the conduit and to minimize the outflow of cells and growth factors out of the structure; furthermore, they should possess adequate mechanical properties to allow its manipulation during surgery and be flexible and soft enough not to compress the regenerated axons $[6,9]$. 
Here we present conduits made from hyaluronic acid (HA), a natural biomaterial that is widely present in the extracellular matrix of most tissues [10]. HA is a non-branched glycosaminoglycan formed by repetitions of D-glucuronic acid and D-Nacetylglucosamine [10]. HA has an excellent biocompatibility and unique biological properties, as it regulates the immune and inflammatory response, the cell differentiation, the vascularization, the scar tissue formation and the cell adhesion to extracellular matrix (ECM) proteins $[10,11]$. In addition, HA modulates the behaviour of glial and immune cells [10]. A very important characteristic of HA is its high hydrophilicity, due to hydrogen bonding interactions between its chains and water $[12,13]$. Scaffolds made out of HA have been studied for the regeneration of the CNS, showing their biocompatibility and ability to induce neural regeneration [14-18].

Tubular scaffolds made out of HA are able to induce seeded Schwann cells to form highly organized structures in vitro $[19,20]$. The HA tubes acted as a template in organizing the growth of the Schwann cells into cylindrical cell sheets. To improve the properties of such biohybrids (structures composed by NGCs with cells) and their biological response here we incorporate Bombyx mori silk fibroin (SF) in the production process of the scaffolds. SF is the term conventionally used for referring to the heavy chain protein which is the main constituent of the silk fibers of the cocoons [21]. SF is a protein with a molecular weight in excess of $300 \mathrm{kDa}$ [22] that presents the specific motif of sequence -GAGAGS-. Due to its biological function, SF has robust mechanical properties and, in addition, a notable biocompatibility [23] and remarkable cell adhesion. This latter property might be favoured by the presence of arginine residues in the sequence of SF. The arginine residues might be recognized by cell membrane adhesion proteins such as integrins and would contribute to the good biological response to the material [22-25]. Besides its excellent in vitro and in vivo biocompatibility SF also shows a slow proteolytic biodegradation [23,26-28]. Combinations of HA and SF have been previously studied as patches for cardiac reparation [29-31], films for growth factor release [32] and dermal tissue regeneration [33], 3D matrices for cartilage tissue engineering [34], and scaffolds for osteoarthritis surgery [35], tissue engineering [36-39] and dermal tissue reconstruction [40]. The presence of SF enhances the mechanical properties of the structures and increases the resistance to degradation when compared to those based only on HA $[39,41,42]$, while the presence of HA increases the water retention and the cell growth when compared to scaffolds made only of SF $[38,39,41-43]$. Regarding the methods of combination of HA 
and SF previously studied, these are based on the physical [42], covalent [44] or enzymatic [39] cross-linking of both materials, being the SF the majority component.

The present work aimed at combining SF and HA in a way such that the celltemplating ability of the HA tubes was preserved, while their mechanical properties and biological response were improved. Our interest thus was in combining both materials but with a majority fraction of HA. It may be noted that both HA and SF are materials currently approved by the United States Food and Drug Administration (FDA) for different uses in biomedical applications in humans, particularly in guidance of peripheral nerve regeneration. [45-48].

\section{Materials and methods}

\subsection{SF preparation}

Fibroin was extracted from Bombyx mori cocoons. Initially, the cocoons were degummed in an aqueous $\mathrm{Na}_{2} \mathrm{CO}_{3}$ solution $(0.2 \mathrm{w} / \mathrm{v})$ and heated to $121^{\circ} \mathrm{C}$ in an autoclave (Sturdy SA-252F) for 50 minutes. The degumming process solubilizes the sericin coating that covers the fibroin fibers, so that it can be removed. Fibroin was washed with distilled water for 20 minutes and the washing step was repeated three times. The fibers were allowed to dry overnight and then immersed in a $9.3 \mathrm{M} \mathrm{LiBr}$ solution and introduced in a furnace at $60{ }^{\circ} \mathrm{C}$ for 4 hours, so that a fibroin solution was obtained. After being dissolved, the silk solution was dialyzed against deionized water to remove the $\mathrm{LiBr}$ salt. Dialysis membranes (BioDesign Dialysis Tubing) with a MWCO of 3500 Da were used. Water was changed every 8 hours and the whole process proceeded for 48 hours. After dialysis the fibroin solution was centrifuged at $5000 \mathrm{rpm}$ for 20 minutes at $21^{\circ} \mathrm{C}$ to remove any debris. Finally, the fibroin was lyophilized and stored in a freezer at $-20{ }^{\circ} \mathrm{C}$.

\subsection{HA and HA-SF films preparation}

A $5 \% \mathrm{w} / \mathrm{w}$ solution of HA derived from Streptococcus equi (1.5-1.8 MDa, 53747, Sigma-Aldrich) in $\mathrm{NaOH} 0.2 \mathrm{M}$ was elaborated by gently stirring for 24 hours under normal conditions of temperature and pressure (NCTP, $\left.25{ }^{\circ} \mathrm{C}, 100 \mathrm{kPa}\right)$. The solution 
was then homogenized by agitation at NCTP for 20 minutes and mixed with the divinyl sulfone cross-linker (DVS; V3700, Sigma-Aldrich) in a 9:10 DVS:HA monomeric units molar ratio. This mixture (hereinafter referred to as HA) was kept under stirring for 10 seconds to achieve the correct mixing and to begin the crosslinking process.

The elaboration of biomaterials based on the combination of HA and SF is analogous to that of HA, with the exception that, after the elaboration of the HA solution, it was mixed with $\mathrm{SF}$ in a concentration of $3 \% \mathrm{w} / \mathrm{v}$ (hereinafter referred to as HA-SF). The process was then continued by homogenization of the solution by stirring for 20 minutes and cross-linking with DVS, as it was done for HA.

Then, the material was injected by pipetting into a polytetrafluoroethylene (PTFE) mold formed by two flat faces on both sides of a U-shaped intermediate plate. After the injection of the material into the mold, it was left for 1 hour in NCTP to achieve the complete crosslinking of the material. Afterwards, it was frozen for 4 hours at $-20{ }^{\circ} \mathrm{C}$ and then for 24 hours at $-80^{\circ} \mathrm{C}$. Once frozen, the material was lyophilized (LyoQuest85, Telstar Life Science) for 24 hours at $20 \mathrm{~Pa}$ and $-80^{\circ} \mathrm{C}$. Finally, the different parts of the mold were separated to proceed to the extraction of the film.

\subsection{HA and HA-SF conduits preparation}

To obtain the consuits, the biomaterials obtained as described in 2.1.2 were injected by pipetting into a PTFE mold formed by elongated channels with a square profile of $2 \mathrm{~mm}$ per side. A polycaprolactone fiber (PCL; Polysciences) with a diameter between 950 and $1050 \mu \mathrm{m}$ was introduced into the center of each channel to form the lumen of the conduit. At both ends of the PCL fiber, a PTFE sheath was inserted with a thickness slightly greater than $2 \mathrm{~mm}$. By pressing the ends of the fiber it was fixed in the center of the channel at the same approximate distance from all the walls. After the injection of the material into the mold, it was left for 10 minutes in NCTP to achieve the complete crosslinking of the material. Subsequently, it was subjected to freezing and lyophilization with the same parameters used for films. Finally, the PCL fibers were removed from the inside, forming the lumen of the conduit.

To study the effect of the dissolution of SF in a basic medium, unprocessed SF (hereinafter referred to as untreated SF) was compared with a $3 \% \mathrm{w} / \mathrm{v}$ SF solution in deionized water (hereinafter referred to as water-treated SF) and with a $3 \% \mathrm{w} / \mathrm{v}$ SF 
solution in $\mathrm{NaOH} 0.2 \mathrm{M}$ (hereinafter referred to as $\mathrm{NaOH}$-treated $\mathrm{SF}$ ). The solutions were homogenized by stirring in NCTP for 20 minutes and then they were spilled into a PTFE plate to give them a film shape. They were then frozen and lyophilized with the same parameters as the other films.

\subsection{Morphology characterization}

For the characterization of the surface morphology and of the geometry of axial and cross-sectional cuts of conduits, a field emission scanning electron microscope (FESEM; ULTRA 55, ZEISS Oxford Instruments) was used. The preparation of the samples consisted primarily in a desiccation under vacuum conditions during the 24 hours prior to the test to avoid interferences due to evaporated water. Subsequently samples were placed on a carbon tape and a carbon bridge was created between the sample and the carbon tape. Finally, samples were coated with a thin layer of platinum. Conduits were cut longitudinally and transversally to observe the interior of the lumen and the morphology of the porosity of the walls. The voltage used was $2 \mathrm{kV}$. The study was performed with three different samples $(n=3)$ made of HA and HA-SF.

\subsection{Elemental analysis}

The FESEM was also used to carry out an elemental analysis through an energy dispersive X-ray spectroscopy (EDS) to verify the presence of SF, by studying the nitrogen $(\mathrm{N})$ content. Samples were prepared as above. The elemental analysis provided the mass fraction $(\omega)$ of $N$, oxygen $(O)$ and sulfur $(S)$ in each selected area of the material. The voltage used was $2 \mathrm{kV}$. The study was performed with three different samples $(n=3)$ of films made of HA and HA-SF.

\subsection{Stability against swelling and drying cycles}

This study was carried out to evaluate the possible loss of material in cycles of swelling and drying. For this purpose, both the amount of non-crosslinked mass and the water uptake were studied. Disc-shaped films of $8 \mathrm{~mm}$ dry diameter $\left(\mathrm{D}_{0}\right)$ of HA and HA-SF were used. Samples were first vacuum desiccated for 6 hours at $50^{\circ} \mathrm{C}$. They 
To study the swelling characteristics, conduits dimensions were measured before and after the absorption of water. For this purpose, conduits made of HA and HA-SF were prepared with two different lengths: samples of approximately $4 \mathrm{~mm}$ in length to study the change in length and samples of approximately $1 \mathrm{~mm}$ in length to study changes in the lumen diameter. Samples were examined and photographed using a binocular magnifying glass (MZ APO, Leica Microsystems). Once images of dry samples were acquired, they were swollen in deionized water for 24 hours and images in swelling state were acquired. The measurement of the dimensions was performed using the ImageJ/FIJI image processing software [49]. The dimensions (length, lumen diameter) in dry and swollen conditions were calculated for 4 different samples $(n=4)$. The variation of the dimensions of the conduits was calculated by the ratios $\mathrm{L} / \mathrm{L} 0$ (length in swelling state (L) divided by length in dry state (L0)) and D/D0 (lumen diameter in swelling state (D) divided by lumen diameter in dry state (D0)).

\subsection{Density and porosity}

Density and porosity of samples were determined gravimetrically, using a balance (AX205, Mettler-Toledo Inc., sensitivity of $0.01 \mathrm{mg}$ ) equipped with a density measurement kit. First, the weight of the NGC in air $\left(W_{a}\right)$ was measured. Subsequently, 
the sample was immersed in n-octane (n-octane: 412236, Sigma-Aldrich) and placed under vacuum for 30 minutes to replace the air inside the pores by n-octane. After removing the n-octane from the lumen of the conduit with an absorbent paper, the weight of the conduits was measured with n-octane in the pores $\left(W_{p}\right)$. Finally, the NGC with n-octane in the pores was completely submerged in n-octane and the apparent weight of the conduits immersed in n-octane $\left(W_{i}\right)$ was measured. Following Archimedes, the density of the sample without considering the air inside the pores was obtained applying equation (1), were $\rho_{\mathrm{o}}$ is the density of n-octane. The porosity was then calculated applying equation (2). This procedure was performed for 3 samples $(\mathrm{n}=$ 3) of HA and HA-SF.

Density $=\frac{W_{a}}{W_{a}-W_{i}} \cdot \rho_{o}$

Porosity $=\frac{V_{\text {pores }}}{V_{\text {total }}}=\frac{W_{p}-W_{a}}{W_{p}-W_{i}}$

\subsection{Thermogravimetric analysis (TGA)}

A thermogravimetric analyzer (TGA/SDTA 851 Mettler-Toledo operated using the STARexx software) was used to study the thermal degradation and composition of the materials. To study the effect of the basic manufacture conditions on SF, untreated, water-treated and $\mathrm{NaOH}$-treated samples of SF were measured. Samples were first maintained at a temperature of $30{ }^{\circ} \mathrm{C}$ for 2 minutes. Then a heating ramp was applied from $30{ }^{\circ} \mathrm{C}$ to $120{ }^{\circ} \mathrm{C}$ with a heating rate of $10{ }^{\circ} \mathrm{C} / \mathrm{min}$. After that, a preheating stage consisting of maintaining a temperature of $120^{\circ} \mathrm{C}$ for 30 minutes was applied to eliminate all the water content of the sample. Finally, a heating ramp was applied from $120{ }^{\circ} \mathrm{C}$ to $720^{\circ} \mathrm{C}$ with a heating rate of $10{ }^{\circ} \mathrm{C} / \mathrm{min}$. The procedure was performed under a positive nitrogen flow $\left(\mathrm{N}_{2}\right)$ of $20 \mathrm{ml} / \mathrm{min}$ and was repeated for three different samples $(n=3)$ of each of the materials mentioned above. As a result, thermograms in which the mass loss of the sample is represented as a function of temperature were obtained. These curves were normalized dividing through the weight existing at the end point of the preheating plateau. 
Assuming that thermal degradation proceeds in composite HA-SF samples as it would for both pure separate components, an estimate of mass loss in composites is given by equation (3), were $\Delta \mathrm{m}_{\mathrm{HA}}$ and $\Delta \mathrm{m}_{\mathrm{SF}}$ are the experimental curves of pure HA and $\mathrm{SF}$, respectively, and $\omega_{\mathrm{SF}}$ is the mass fraction of SF.

$$
\Delta \mathrm{m}_{\mathrm{comp}}=\omega_{\mathrm{SF}} \cdot \Delta \mathrm{m}_{\mathrm{SF}}+\left(1-\omega_{\mathrm{SF}}\right) \cdot \Delta \mathrm{m}_{\mathrm{HA}}
$$

This equation was employed to deduce a value of $\omega_{\mathrm{SF}}$ from the measured curves of $\Delta \mathrm{m}_{\mathrm{HA}}, \Delta \mathrm{m}_{\mathrm{SF}}, \Delta \mathrm{m}_{\mathrm{exp}}$. For this purpose, $\Delta \mathrm{m}_{\text {comp }}$ given by equation (3) was least-squares fitted to $\Delta \mathrm{m}_{\mathrm{exp}}$ taking $\omega_{\mathrm{SF}}$ as fitting parameter.

\subsection{Characterization of mechanical properties}

The mechanical characterization of the materials was performed by rotational rheometry (Discovery Hybrid Rheometer DHR, TA Instruments) in order to study the effect of incorporating SF on the shear modulus $(\mathrm{G})$ of the material. The samples (circular films $20 \mathrm{~mm}$ in diameter) were studied in a state of swelling in deionized water for 24 hours.

An oscillation test was performed with a frequency of $1 \mathrm{~Hz}$ and torque amplitudes ranging from 10 to $100 \mu \mathrm{N} \cdot \mathrm{m}$ with a logarithmic sweep. Since the samples' diameter, $20 \mathrm{~mm}$, didn't coincide with the diameter of the rheometer plates, $25 \mathrm{~mm}$, the modulus values calculated by the equipment's software were corrected to take this mismatch into account, by multiplying the software's data with the ratio of both areas.

\subsection{Schwann cell culture}

Culture of rat Schwann cells (rSCs; P10301, Innoprot) was performed on $8 \mathrm{~mm}$ diameter films and on NGCs made of HA and HA-SF to study the cell adhesion and proliferation.

A sanitization of the materials (films and conduits) was first carried out by immersion in $70 \%$ ethanol (ET00021000, Scharlab) for 2 hours, in $50 \%$ ethanol for 10 minutes and in $30 \%$ ethanol for another 10 minutes. Thereafter the ethanol residues 
were removed by performing 3 washes of 10 minutes with ultrapure water (Mili-Q ${ }^{\circledR}$ ). The preconditioning of the materials was done by immersion in culture medium (Dulbecco's modified Eagle medium with a high glucose level (4.5 g/L) (DMEM; 21331020, Life Technologies) supplemented with $10 \%$ of fetal bovine serum (FBS; 10270-106/A3381E, Life Technologies) and $1 \%$ penicillin/streptomycin (P/S; 15140122, Life Technologies)) and incubation at $37{ }^{\circ} \mathrm{C}$ for 24 hours in a humidified atmosphere containing $5 \% \mathrm{CO}_{2}$.

After the expansion of the rSCs in a cell culture flask, they were washed with PBS and a trypsin/EDTA solution (T/E; 25200-072, Life Technologies) was then added to break the cell-matrix and cell-cell interactions in order to remove the cells from the bottom of the culture bottle. After centrifugation at $1080 \mathrm{rpm}$ for 5 minutes, the pellet was resuspended in Schwann cell culture medium (P60123, Innoprot). At this moment, the seeding with rSCs in passage 5 was performed with a seeding density of 10.000 cells per film and 250.000 cells per NGC. Finally, the culture was introduced in an incubator at $37^{\circ} \mathrm{C}$ with a humid atmosphere containing $5 \% \mathrm{CO}_{2}$ for 1 and 5 days in the case of films and for 1, 5 and 10 days in the case of NGCs, renewing the Schwann cell culture medium every 48 hours.

\subsection{Fluorescent staining of the cytoskeleton}

The morphologies of the cells were examined by fluorescently staining their F-actin cytoskeletons. Stains were performed on the cultured rSCs to carry out the labelling of specific proteins and observe the cellular arrangement. The following procedure was repeated after 1 and 5 days of culture in the case of films and after 1, 5 and 10 days in the case of conduits.

First, the Schwann cell medium was removed and the materials (films and conduits) were washed with PBS. Thereafter, cells were fixed with $4 \%$ paraformaldehyde (PFA; 47608, Sigma-Aldrich) for 20 minutes at room temperature. After cell fixation, 3 washes of 10 minutes with DPBS were performed to remove PFA residues. At this point, the non-specific bindings were blocked and the cell membrane was permeabilized by the use of a blocking buffer composed of DPBS with $3 \%$ bovine serum albumin (BSA; A7906, Sigma-Aldrich) and $0.1 \%$ Tween20 (P1379, Sigma-Aldrich) for 45 minutes at room temperature. Cells were finally stained with FITC-phalloidin (B607, Life Technologies) to mark the cytoskeleton actin filaments (green colour) and with 
DAPI (D9564, Sigma-Aldrich) at a 1/5000 dilution for 10 minutes to mark the cells nuclei (blue colour). The imaging was performed using a confocal microscope (LEICA TCS SP5, Leica microsystems).

From the images obtained by confocal microscopy, and thanks to staining with FITC-phalloidin, the total area occupied by the cells cytoskeleton was calculated. ImageJ/FIJI image processing software was used for this purpose [49]. Four different samples $(n=4)$ of HA and HA-SF films and conduits were studied.

\subsection{MTS cell proliferation assay}

To evaluate the cell proliferation inside the conduits, MTS assays (CellTiter 96 Aqueous One Solution Cell Proliferation Assay, Promega) were carried out on HA and HA-SF conduits ( $n=3$ each). When incorporated to the cells, the MTS was bioreduced by metabolically active cells at different times of cell culture (1, 5 and 10 days) in a rate proportional to the number of live cells. After 3 hours of incubation with the reagent, the medium was removed and its absorbance was measured with a Victor Multilabel Counter 1420 spectrophotometer (Perkin-Elmer) at $490 \mathrm{~nm}$.

\subsection{Biocompatibility of subcutaneously implanted scaffolds}

All procedures were performed under the Spanish Regulations for animal experimentation (Laws 53/2013, 178/2004) with the approval of Animal Care Committee of the Polytechnic University of Madrid (Madrid, Spain) and according to the ARRIVE (Animal Research: Reporting In Vivo Experiments) guidelines. In vivo studies were conducted in adult male CD-1 mice (35-40 g body weight; 12-15 weeks old; $n=5$ mice for each group). All mice were bred and housed in the animal facility of the Centre for Biomedical Technology. All animals were kept at constant temperature $\left( \pm 22^{\circ} \mathrm{C}\right)$ and humidity $(\sim 52 \%)$ with free access to food and water in 12 hours light/dark cycle.

HA and HA-SF scaffolds $(1.5$ x $6 \mathrm{~mm})$ were subcutaneously implanted at different locations in each mouse to compare the bioresponse of both scaffolds in the same animal and reduce data dispersion due to inter-animal variability. A sanitization of the materials was previously carried out by immersion in $70 \%$ ethanol for 2 hours, in $50 \%$ ethanol for 10 minutes and in $30 \%$ ethanol for another 10 minutes. Afterwards the 
ethanol residues were removed by performing 4 washes of 10 minutes with ultrapure water. The preconditioning of the materials was done by immersion in PBS for 24 hours.

Surgeries were performed under anaesthesia with $2 \%$ Isoflurane in air. The back of each mouse was shaved, and the skin was disinfected with povidone-iodine. All surgeries were made under aseptic conditions and Vaseline was administrated to protect the eyes from dehydration. To implant the scaffolds, an incision of approximately $1 \mathrm{~cm}$ was made on the dorsal part of each mouse and the subcutaneous space was separated with scissors. The incisions were then sutured with 3-0 surgical Nylon. Buprenorphine $(0.05-0.1 \mathrm{mg} / \mathrm{kg})$ was administered as a pain reliever for 48 hours post-surgery and, if signs of pain were seen, Ibuprofen $(30 \mathrm{mg} / \mathrm{kg}$ ) was dispensed via oral in drinking water for 1 week. All animals were then carefully monitored for the following 3 days by animal care services. Sham-operated animals as negative controls were subjected to identical procedures, but no scaffolds were implanted in this case.

At different time points after scaffolds implantation (1, 4 and 8 weeks), the mice were euthanized in $100 \% \mathrm{CO}_{2}$ atmosphere (5-10 $\mathrm{min}$ ) followed by cervical dislocation. The dorsal skin was carefully resected and immediately immersed in PBS solution. The skin sections containing the scaffolds together with the surrounding tissues were excised and fixed in 4\% paraformaldehyde for 7 days. Before cutting, skin sections were cryoprotected in 30\% sucrose. Samples were embedded in optimal cutting temperature compound and serial $10 \mu \mathrm{m}$ thick sections were cut with a Microm HM550 cryostat (Thermo Scientific, Kalamazoo, USA). Cryostat sections were stained with hematoxylin-eosin (H\&E) and Masson's trichrome. To examine cell infiltration, extracellular matrix deposition and neovascularisation, micrographs were captured through an Olympus BX51 microscope (Olympus DP70, Olympus America Inc. Center Valley, PA, EEUU).

\subsection{Statistical analysis}

Results were expressed as mean \pm standard deviation (SD). The statistical analysis of the results was performed with the software GraphPad Prism 6 using the non-parametric Mann-Whitney test based on rank comparison. Statistically significant differences are indicated by an asterisk (*), indicating a p-value below 0.05 . Four asterisks $(* * * *)$ indicate a p-value below 0.0001 . 


\section{Results and discussion}

\subsection{Effect of preparation procedures on material composition}

The crosslinking reaction of HA with DVS takes place in basic $\mathrm{pH}$ [10]. Since an aqueous solution of SF was mixed with the basic HA-DVS solution during the material preparation process, it was necessary to assess the effect of exposure of SF to the basic solution. A thermogravimetric analysis (TGA) of untreated SF, water-treated SF and $\mathrm{NaOH}$-treated SF (Fig. 1A) shows slight modifications of the temperature-dependent mass loss. Prolonged exposure of SF to a basic medium causes hydrolysis of peptide bonds [50]. Therefore, when SF is added to the solution of $5 \% \mathrm{HA}$ in $0.2 \mathrm{M} \mathrm{NaOH}$, it is subjected to a basic medium throughout the process and it suffers the hydrolysis of a number of peptide bonds. Chemical alteration of silk by immersion in a $\mathrm{NaOH}$ solution accumulates to the reduction in the molecular weight induced by the degumming process. It was found that this reduction is larger when $\mathrm{Na}_{2} \mathrm{CO}_{3}$ is added to the degumming medium due to the resulting $\mathrm{pH}$ of the solution [51]. TGA data reflects an alteration as a difference in both curves in the range between $500{ }^{\circ} \mathrm{C}$ and $700{ }^{\circ} \mathrm{C}$. Fig. 1B shows the thermograms of untreated HA, NaOH-treated SF and of HA-SF films. The composite HA-SF materials have degradation patterns somehow intermediate, as expected. With these data, an estimate of the SF mass fraction in the composite sample can be obtained by least-square fitting the parameter in equation (3), as explained above. This gives a figure of $\boldsymbol{\omega}_{\mathbf{S F}, \mathbf{e x p}}=37 \pm 5$, close enough to the theoretical $\boldsymbol{\omega}_{\mathbf{S F}, \text { th }}=$ $37.50 \pm 0.01$, confirming efficient incorporation of SF to the HA matrix during the manufacturing process.

The different $\mathrm{N}$ content of SF and HA is reflected in the EDS data in Table 1. Theoretically, the mass fractions of $\mathrm{N}\left(\boldsymbol{\omega}_{\mathbf{N}}\right)$ in HA and in HA-SF samples are $2.60 \%$ and $6.75 \%$, respectively. As shown in Table 1, the experimental mass fractions of $\mathrm{N}$ were lower than the theoretical ones. It should be noted that the elemental analysis is performed on very small areas of material, so a non-homogeneous distribution of SF could produce this difference. In addition, elemental analysis by EDS is sensitive solely to surface composition. Table 1 shows also the ratios between $\boldsymbol{\omega}_{\mathbf{N}}$ and the mass fractions of $\mathrm{O}$ and $\mathrm{S}$. All these parameters were substantially higher in the HA-SF samples than in the HA samples, proving the presence of SF in the matrices. 


\subsection{Stability against swelling and drying cycles}

The loss of mass of HA and HA-SF films after prolonged immersion in water was analysed to determine the stability against solution of the SF incorporated to the HA matrix, and whether the presence of SF could alter the sol fraction of HA in the crosslinked gel. As seen on Table 1, there were no significant differences in the mass loss between HA and HA-SF films after being swollen in water. A similar sol fraction (around $30 \%$ ) obtained in both systems.

Dimensional change upon swelling was expressed by computing the ratio of measured diameters of discs after swelling (D) and dry $\left(\mathrm{D}_{0}\right)$. Though small, the effect of SF is to constrain swelling and dimensional change, as expected (Table 1).

\begin{tabular}{lll}
\hline & HA & HA-SF \\
\hline$\omega_{\mathrm{N}_{\exp }}[\%]$ & $1.0 \pm 0.1$ & $3.8 \pm 0.9$ \\
$\omega_{\mathrm{N}} / \omega_{0}$ & $0.04 \pm 0.01$ & $0.20 \pm 0.01$ \\
$\omega_{\mathrm{N}} / \omega_{\mathrm{S}}$ & $0.18 \pm 0.03$ & $1.2 \pm 0.2$ \\
$\mathrm{~m} / \mathrm{m}_{0}$ & $0.72 \pm 0.01$ & $0.70 \pm 0.01$ \\
$\mathrm{D} / \mathrm{D}_{0}(1 \mathrm{st}$ swelling $)$ & $2.0 \pm 0.1$ & $1.78 \pm 0.06$ \\
$\mathrm{D} / \mathrm{D}_{0}(2 \mathrm{nd}$ swelling) & $2.1 \pm 0.1$ & $1.92 \pm 0.09$ \\
$\mathrm{Q}(1 \mathrm{st}$ swelling) & $8 \pm 1$ & $5.7 \pm 0.6$ \\
$\mathrm{Q}(2 \mathrm{nd}$ swelling) & $9 \pm 2$ & $7 \pm 1$ \\
\hline
\end{tabular}

Table 1. Physicochemical properties of HA and HA-SF films. The quantities are defined in the text. Mass fraction from elemental analysis ( $\boldsymbol{\omega})$; swelling parameters: mass $\left(\mathrm{m} / \mathrm{m}_{0}\right)$, diameter $\left(\mathrm{D} / \mathrm{D}_{0}\right)$ and swelling $(\mathrm{Q})$ ratios. Values are expressed as mean $\pm \mathrm{SD}$.

\subsection{Mechanical properties of HA-SF}


The mechanical behaviour of HA and HA-SF discs was assessed in shear experiments, in a rotational rheometer. Fig. 2A shows the storage modulus ( $\mathrm{G}^{\prime}$, the real part of the complex shear modulus) obtained as a function of the oscillatory torque. $\mathrm{G}^{\prime}$ quantifies the recoverable energy in a cycle, and thus measures the elasticity of the material. There is a clear increase of $\mathrm{G}^{\prime}$ in samples containing SF, practically doubling the $G^{\prime}$ value for all the oscillation torques. This implies that the addition of SF allows the material to store more energy without suffering permanent deformation, indicating that interactions between SF and HA molecules make the HA chains less pliable to deformation, resulting in a greater stiffness of the material. There is a slight decrease in $\mathrm{G}^{\prime}$ when the magnitude of the applied torque increases. This non-linear behaviour of the material is indicative of strain-softening, and may be due to the rupture of SF $\cdots \mathrm{HA}$ and HA ‥HA intermolecular interactions with larger values of torque. The loss modulus (G", the imaginary part of the complex shear modulus) is shown on Fig. 2B. G" is a measure of the non-recoverable energy in a cycle due to different dissipative processes, and characterizes the viscous component of the material's response. Large values of G" are typical of soft hydrogels, and originate in internal friction due to water diffusion and chain slippage during deformation. Presence of SF in the material results in an increase of G", especially for low torque amplitudes, where the increase is almost double. This is indicative of the occurrence of new, additional frictional processes in the medium, arising from the SF $\cdots \mathrm{HA}$ interactions as relative slippage and as formation and diffusion of defects. When the oscillation torque exceeds $40 \mu \mathrm{N} \cdot \mathrm{m}$, the difference decreases considerably. The fact that this difference is sensibly higher at lower deformations may suggest that it be related to the diffusion of water molecules in both gels, which is altered in the presence of the protein molecules. As already commented, the stiffness of the HA-SF material, as characterized by $\mathrm{G}^{\prime}$, is greater than that of HA; however, its viscoelasticity, as characterized by the ratio $\mathrm{G}^{\prime} / \mathrm{G}^{\prime \prime}$, doesn't change very much (see Table $2)$.

\begin{tabular}{lllll}
\hline & HA & HA-SF \\
\cline { 2 - 5 } & & \multicolumn{4}{c}{$10 \mu N \cdot m$} & $100 \mu N \cdot m$ & $100 \mu N \cdot m$ \\
\hline $\mathrm{G}^{\prime} / \mathrm{G}^{\prime \prime}$ & $6.8 \pm 0.7$ & $6 \pm 1$ & $7 \pm 2$ & $7 \pm 2$ \\
\hline
\end{tabular}


Table 2. G'/G" ratios for HA and HA-SF at two different torque values, $10 \mu \mathrm{N} \cdot \mathrm{m}$ and $100 \mu \mathrm{N} \cdot \mathrm{m}$. Values are expressed as mean $\pm \mathrm{SD}$.

\subsection{Adhesion and proliferation of Schwann cells on HA-SF films}

Rat Schwann cells (rSCs) were seeded and cultured for 1 and 5 days on HA and HASF films in order to assess differences in adherence and proliferation. For the visualization of the cells by confocal microscopy, a staining process was performed with FITC-phalloidin 488 to visualize their cytoskeletal actin fibers (green color) and with DAPI to visualize the cell nucleus (blue color). For the day 1, Fig. 3A and 3C show representative images of both studied cases in order to appreciate the differences in the total extent of the cytoskeleton, which is indicative of the cell adhesion characteristics on the material. On HA substrates (Fig. 3A) early cell adhesion was scarce, and the cells had a marked globular morphology, in conformity with known facts [52]. This is a consequence of the highly hydrophilic nature of HA: the enormous amounts of water molecules which surround the HA chains hinder ECM protein deposition on its surface and thus difficult cell adhesion. This means that cell adhesion on the surface of HA materials must rely primarily on physical phenomena such as the surface roughness, where differential accumulation of HA molecules offers more stable anchoring sites to ECM proteins. With the material containing SF (Fig. 3C) early cell adhesion was clearly greater, with the cells exhibiting a much more developed cytoskeleton. Several studies have shown that SF has good cell adhesion properties $[25,53]$. Although the RGD (Arg-Gly-Asp) cell binding motif as such is not present in the aminoacid sequence of Bombyx mori SF, an amino acid sequence has been observed in a non-repetitive C-terminal region with a large number of basic amino acids, especially arginine, which play a key role in the cell adhesion properties of SF [25]. Arginine residues can be recognized by transmembrane cell adhesion proteins such as integrins, which mediate in the interaction between cell and substrate $[54,55]$.

In order to quantify somehow the extent of cell adhesion, the total area occupied by the cytoskeleton of the cells was measured (Fig. 3E), confirming the conclusions obtained in a qualitative way. It can be seen that early cell adhesion in HA films is very low, while addition of SF clearly enhances early cell adhesion.

The same features were analysed after 5 days of cell culture (Fig. 3B and 3D) in order to compare early with late cell adhesion characteristics. On HA films cells now 
exhibited an elongated shape (arrows in Fig. 3B). Some cells have been able to attach to specific sites on the material surface due to mediated cell $\cdots \mathrm{HA}$ interactions, but preferentially the cells entertain cell $\cdots$ cell bonds forming filamentary structures with the shape of a necklace. In contrast, cells cultured on films with SF (Fig. 3D) show larger fibrillary cytoskeleta spread on the material, indicative of a greater cell $\cdots$ surface interaction. When quantified (Fig. 3E), this conclusion is further reinforced.

\subsection{Morphology and physicochemical properties of conduits}

Longitudinal (axial) and transverse cuts of conduits show the lumina of the conduits and the porous walls, Fig. 4. Both in HA and HA-SF conduits the lumen surface is always tighter and less porous than the walls, which is important to ensure that the cells seeded within the lumen are retained inside the conduit and cannot protrude across its walls. This is due to the fact that the surface of the PCL fiber used as a template of the lumen is hydrophobic; thus, in its vicinity non-swollen HA chains are predominant $[19,20]$. Water crystallization and subsequent lyophilization then produce a thin layer of denser HA close to the lumen. The pores observed in the conduit's wall matrix are produced by the phase-separation of water during the crystallization process. This structure ensures good diffusion of molecules across the conduit (water, oxygen, nutrients, factors), while efficiently retains the cells seeded inside the tube. The addition of SF to the HA matrix of the conduits thus didn't modify the main features of the original HA conduits. Fig. 4D' shows a detail of the internal surface of the HA-SF conduit, where elongated structures arranged on the surface (indicated with arrows) could correspond to SF fibers.

The swelling behaviour of HA and HA-SF conduits was characterized by the change in dimensions of conduit length and lumen diameter, Table 3 . There is no significant difference in dimensional change between HA and HA-SF samples. Though very small, the effect of SF seems to be to introduce some anisotropy in the conduit's swelling as compared with the HA tubes: the HA-SF tubes swell somewhat less than the HA tubes transversally to their axis, while they swell somewhat more axially. This may have to do with anisotropies of the SF fibril distribution induced during the phase separation of crystallized water, a process which is determined by the geometry of the heat transfer between gel and mold during cooling. 
Table 3 shows the density and porosity of conduits made of HA and HA-SF. Regarding the density values, there's a slight decrease for HA-SF conduits, but it is not enough to show a statistical difference. This slight decrease may be due to the incorporation of SF, since SF has a lower density than HA [56,57]. Regarding the porosity values, the slight differences are not significant. This could be expected, since the pores are produced by the crystallization of the large amounts of water present in the samples, which are insignificantly influenced by the presence of the SF molecules.

\begin{tabular}{lll}
\hline & HA & HA-SF \\
\hline $\mathrm{L} / \mathrm{L}_{0}$ & $1.41 \pm 0.06$ & $1.6 \pm 0.2$ \\
$\mathrm{D}_{\text {lumen }} / \mathrm{D}_{\text {lumen }_{0}}$ & $1.21 \pm 0.06$ & $1.11 \pm 0.05$ \\
Density $\left[\mathrm{g} \mathrm{cm}^{-3}\right]$ & $1.22 \pm 0.08$ & $1.1 \pm 0.1$ \\
Porosity $[\%]$ & $79 \pm 1$ & $78 \pm 5$ \\
\hline
\end{tabular}

Table 3. Physicochemical properties of HA and HA-SF conduits. Density was calculated by equation (1) and porosity by equation (2). Swelling ratios L/L0 (length) and D/D0 (diameter) are explained in the text. Values are expressed as mean \pm SD.

\subsection{HA-SF-Schwann cells biohybrids}

Schwann cells seeded within the lumen of HA tubular scaffolds create a continuous cylinder which adapts to the inner channel surface, which acts as a template for the formation of such a cell sheath $[19,20]$. In HA tubes this process needs 9 days, and it is the result of the interplay of cell proliferation and cell $\cdots$ cell contacts being preferred over cell $\cdots$ material contacts, due to the highly hydrophilic nature of the HA matrix. To study this process in HA-SF tubes rSCs were seeded and cultured for 1, 5 and 10 days. Staining with FITC-phalloidin and DAPI permit to visualize the cell cytoskeleton (green in Fig. 5) and nuclei (blue), respectively. A comparison of Fig. 5A and 5D shows that at day 1 there are already differences between both materials, cell density and spread being higher in the HA-SF tubes. At day 5 (Fig. 5B and 5E) considerable proliferation of SCs has occurred in both cases. However, while the 
number of cells inside the HA conduit is still not enough to form the rSCs sheath, in the case of the HA-SF conduit the cell population is already organized as a cell cylinder (arrows in Fig. 5E indicate how this sheath folds). This indicates that the higher cell adhesion and proliferation obtained by the incorporation of SF to the conduits allows us to obtain the rSCs sheath earlier when compared with NGCs based solely on HA. After 10 days culture the cell sheath is fully developed in both scaffolds, Fig. 5C and 5F. Previous studies showed that this kind of cell structure took 9 days to develop in HA tubular scaffolds $[19,20]$. We now see that the presence of SF protein in the HA matrix preserves the templating ability of the scaffold channel to induce the formation of the cell cylinder and accelerates it. Thus, while SF significantly increases cell adhesion on the channel surface due to the supply by SF of additional binding motifs, still cell $\cdots$ cell binding is preferred over cell $\cdots$ surface binding, as revealed by the easiness with which the cell cylinder gets unstuck from the scaffold (Fig. 5E and 5F).

A MTS assay performed after 1, 5 and 10 days of cell culture (Fig. 6) shows that the presence of SF significantly translates into higher cell proliferation inside the conduits, explaining the earlier formation of the cell sheath in the HA-SF conduits.

\subsection{Biocompatibility of subcutaneously implanted biomaterials}

During the study there were no cases of pain, inflammation or infection caused by the implantation of scaffolds. The skin sections containing the scaffolds were photographed at 1, 4 and 8 weeks after their implantation (Fig. 7). As can be seen in Fig. 7, the scaffolds are surrounded by healthy tissue since the first week. The presence of blood vessels near to or in direct contact with the scaffolds is also always visible (white arrows in Fig. 7).

Biocompatibility of subcutaneously implanted biomaterials was assessed by H\&E and Masson's trichrome staining of fixed scaffolds after its implantation for 1, 4 and 8 weeks (Fig. 8 for HA scaffolds and Fig. 9 for HA-SF scaffolds). An overall view of a representative longitudinal section of each scaffold is shown in first place (A, E and I for H\&E staining; C, G and K for Masson's trichrome staining), and a magnified view of the perimeter of each biomaterial is shown in second place $(B, F$ and $J$ for $H \& E$ staining; D, H and L for Masson's trichrome staining), at each post-implantation time point. 
One week after the scaffold implantation, a moderate immune response can be observed for both HA (Fig. 8 A-D) and HA-SF (Fig. 9 A-D) scaffolds. In both cases a fibrous capsule surrounding the scaffold is clearly visible, which is a typical reaction after the implantation of a biomaterial. There is also a dense population of granulocytes surrounding the scaffolds. Notably, some collagen structures and some cross sections of blood vessels and capillaries are present, although small and isolated.

After 4 weeks, a reduction of the immune response can be observed, corresponding to a mild foreign body reaction for both HA (Fig. 8 E-H) and HA-SF (Fig. 9 E-H) scaffolds. The population of granulocytes has been reduced and it has infiltrated inside the scaffold. Now, a greater presence of macrophages is observable, which indicates that the bioresorption process of the scaffold has begun. Remarkably, there is an increase of the number and extension of collagen structures, indicating that new extracellular matrix is being deposited. In addition, more cross sections of blood vessels and capillaries and more red blood cells are observable around and inside the scaffold. It seems that this process of collagen deposition and vascularization inside the scaffold is accelerated when SF is present, since more collagen structures and more blood vessels can be observed inside the scaffold.

Finally, after 8 weeks, the immune response has been drastically reduced, so both HA (Fig. 8 I-L) and HA-SF (Fig. 9 I-L) scaffolds have been completely resorbed. The fibrous capsule has disappeared and the population of granulocytes and macrophages at the scaffold site has almost disappeared, with the epidermis tissue surrounding the scaffold appearing normal. There is a clear increase of the deposition of collagen structures around and inside the cavities of the scaffolds, compared with week 4, and individual collagen fibres are clearly visible within the collagen matrix (Fig. 10). This increased presence of collagen structures is associated to the presence of active fibroblasts (spindle shaped cells in Fig. $10 \mathrm{~B}$ and Fig. $10 \mathrm{D}$ for HA and HA-SF scaffolds, respectively), that can be observed infiltrating into the scaffold from the surrounding epidermis. Regarding the vascularization process, it is quite similar to the one observed after 4 weeks. Multiple cross sections of blood vessels and capillaries are observed around and inside the scaffold, as well as populations of isolated blood cells (Fig. 10). At this time there is not a notably difference between HA and HA-SF scaffolds regarding the collagen deposition and the vascularization. 
These results suggest that both HA and HA-SF scaffolds are biointegrated by the host after 8 weeks of implantation and that, furthermore, the processes of extracellular matrix deposition and angiogenesis are accelerated when SF is present.

\section{Conclusions}

Silk fibroin protein can be efficiently incorporated into the manufacturing process of HA-based tubular scaffolds intended as nerve conduits. The developed materials are stable over time after swelling and drying cycles. SF supplies cell-binding sequences which lead to higher Schwann cell adhesion and proliferation in the HA-SF materials when compared with the HA ones. While most physicochemical properties (density, porosity and swelling degree) of both materials are indistinguishable, manipulability and mechanical shear modulus are improved by the presence of SF. The HA-SF tubular scaffolds can act as templates of Schwann-cell macrocylinders, as had the HA scaffolds, but the process is significantly accelerated in HA-SF scaffolds. The biocompatibility of both HA and HA-SF scaffolds has been proven, and both biomaterials were accepted by the host after 8 weeks of implantation. This finding is of importance when thinking of the HA-SF-Schwann cells constructs as transplantable biohybrids, since it considerably shortens the in vitro and in vivo strategies, where tubulisation techniques are required for regeneration.

\section{Conflicts of interest}

There are no conflicts to declare.

\section{Acknowledgements}

The authors acknowledge financing from the Spanish Ministry of Economy and Competitiveness through grants RTI2018-095872-B-C22/ERDF, DPI2015-72863-EXP, MAT2016-79832-R, MAT2016-76847-R and, and community of Madrid through grant Neurocentro-B2017/BMD-3760. FGR acknowledges scholarship FPU16/01833 of the Spanish Ministry of Education, Culture and Sports. We thank the Electron Microscopy Service at the UPV, where the FESEM images were obtained.

\section{References}

[1] E. a Huebner, S.M. Strittmatter, Axon Regeneration in the Peripheral and Central 
Nervous Systems, Results Probl. Cell Differ. Author Manuscr. 48 (2009) 339351. doi:10.1007/400.

[2] J.W. Fawcett, R.A. Asher, The glial scar and central nervous system repair, Brain Res. Bull. 49 (1999) 377-391. doi:10.1016/S0361-9230(99)00072-6.

[3] A.H. Koeppen, Wallerian degeneration: History and clinical significance, J. Neurol. Sci. 220 (2004) 115-117. doi:10.1016/j.jns.2004.03.008.

[4] S. Hall, The response to injury in the peripheral nervous system., J. Bone Jt. Surg. 87 (2005) 1309-19. doi:10.1302/0301-620X.87B10.16700.

[5] P. Dubový, I. Klusáková, I. Hradilová Svíženská, Inflammatory profiling of Schwann cells in contact with growing axons distal to nerve injury, Biomed Res. Int. 2014 (2014). doi:10.1155/2014/691041.

[6] K.S. Houschyar, A. Momeni, M.N. Pyles, J.Y. Cha, Z.N. Maan, D. Duscher, O.S. Jew, F. Siemers, J. van Schoonhoven, K.S. Houschyar, A. Momeni, M.N. Pyles, J.Y. Cha, Z.N. Maan, D. Duscher, O.S. Jew, F. Siemers, J. van Schoonhoven, The Role of Current Techniques and Concepts in Peripheral Nerve Repair, Plast. Surg. Int. 2016 (2016) 1-8. doi:10.1155/2016/4175293.

[7] L. Tian, M.P. Prabhakaran, S. Ramakrishna, Strategies for regeneration of components of nervous system: scaffolds, cells and biomolecules., Regen. Biomater. 2 (2015) 31-45. doi:10.1093/rb/rbu017.

[8] A. Muheremu, Q. Ao, Past, Present, and Future of Nerve Conduits in the Treatment of Peripheral Nerve Injury, 2015 (2015). doi:10.1155/2015/237507.

[9] S. Kehoe, X.F. Zhang, D. Boyd, FDA approved guidance conduits and wraps for peripheral nerve injury: A review of materials and efficacy, Injury. 43 (2012) 553-572. doi:10.1016/j.injury.2010.12.030.

[10] H.G. Garg, C.A. Hales, Chemistry and biology of hyaluronan, Elsevier, 2004. https://books.google.es/books?hl=es\&lr=\&id=WCjv3_q2ggC\&oi=fnd\&pg=PP1\&dq=chemistry + and + biology + of + hyaluronan $\&$ ots $=F S$ m4Y8GDCu\&sig=QdBL61rlFZbNjhWMvoX7xVZX6AU\#v=onepage\&q=chemi stry and biology of hyaluronan\&f=false (accessed February 4, 2018).

[11] M.N. Collins, C. Birkinshaw, Hyaluronic acid based scaffolds for tissue engineering - A review, Carbohydr. Polym. 92 (2013) 1262-1279. doi:10.1016/j.carbpol.2012.10.028.

[12] J.E. Scott, Secondary structures in hyaluronan solutions: chemical and biological implications., Ciba Found. Symp. 143 (1989) 6-15; discussion 15-20, 281-5. 
http://www.ncbi.nlm.nih.gov/pubmed/2680349 (accessed June 4, 2017).

[13] M.K. Cowman, S. Matsuoka, Experimental approaches to hyaluronan structure, Carbohydr. Res. 340 (2005) 791-809. doi:10.1016/j.carres.2005.01.022.

[14] Y. Liang, P. Walczak, J.W.M. Bulte, The survival of engrafted neural stem cells within hyaluronic acid hydrogels, Biomaterials. 34 (2013) 5521-5529. doi:10.1016/j.biomaterials.2013.03.095.

[15] T.-W. Wang, M. Spector, Development of hyaluronic acid-based scaffolds for brain tissue engineering., Acta Biomater. 5 (2009) 2371-84. doi:10.1016/j.actbio.2009.03.033.

[16] J. Ma, W.-M. Tian, S.-P. Hou, Q.-Y. Xu, M. Spector, F.-Z. Cui, An experimental test of stroke recovery by implanting a hyaluronic acid hydrogel carrying a Nogo receptor antibody in a rat model., Biomed. Mater. 2 (2007) 233-40. doi:10.1088/1748-6041/2/4/005.

[17] W.M. Tian, S.P. Hou, J. Ma, C.L. Zhang, Q.Y. Xu, I.S. Lee, H.D. Li, M. Spector, F.Z. Cui, Hyaluronic acid-poly-D-lysine-based three-dimensional hydrogel for traumatic brain injury., Tissue Eng. $11 \quad$ (2005) 513-25. doi:10.1089/ten.2005.11.513.

[18] M. Pérez-Garnes, J.A. Barcia, U. Gómez-Pinedo, M. Monleón Pradas, A. VallésLluch, Materials for Central Nervous System Tissue Engineering, in: Cells Biomater. Regen. Med., InTech, 2014. doi:10.5772/59339.

[19] G. Vilariño-Feltrer, C. Martínez-Ramos, A. Monleón-De-La-Fuente, A. VallésLluch, D. Moratal, J.A. Barcia Albacar, M. Monleón Pradas, Schwann-cell cylinders grown inside hyaluronic-acid tubular scaffolds with gradient porosity, Acta Biomater. 30 (2016) 199-211. doi:10.1016/j.actbio.2015.10.040.

[20] I. Ortuno-Lizarán, G. Vilarino-Feltrer, C. Martinez-Ramos, M.M. Pradas, A. Vallés-Lluch, Influence of synthesis parameters on hyaluronic acid hydrogels intended as nerve conduits, Biofabrication. 8 (2016). doi:10.1088/17585090/8/4/045011.

[21] R. J. Lancashire, Chemistry of Garments: Animal Fibres, Dep. Chem. Univ. West Indies.

(2011). http://wwwchem.uwimona.edu.jm/courses/CHEM2402/Textiles/Animal_Fibres.h tml (accessed June 3, 2017).

[22] Q. Xia, Z. Zhou, C. Lu, D. Cheng, F. Dai, B. Li, P. Zhao, X. Zha, T. Cheng, C. Chai, G. Pan, J. Xu, C. Liu, Y. Lin, J. Qian, Y. Hou, Z. Wu, G. Li, M. Pan, C. Li, 
Y. Shen, X. Lan, L. Yuan, T. Li, H. Xu, G. Yang, Y. Wan, Y. Zhu, M. Yu, W. Shen, D. Wu, Z. Xiang, J. Yu, J. Wang, R. Li, J. Shi, H. Li, G. Li, J. Su, X. Wang, G. Li, Z. Zhang, Q. Wu, J. Li, Q. Zhang, N. Wei, J. Xu, H. Sun, L. Dong, D. Liu, S. Zhao, X. Zhao, Q. Meng, F. Lan, X. Huang, Y. Li, L. Fang, C. Li, D. Li, Y. Sun, Z. Zhang, Z. Yang, Y. Huang, Y. Xi, Q. Qi, D. He, H. Huang, X. Zhang, Z. Wang, W. Li, Y. Cao, Y. Yu, H. Yu, J. Li, J. Ye, H. Chen, Y. Zhou, B. Liu, J. Wang, J. Ye, H. Ji, S. Li, P. Ni, J. Zhang, Y. Zhang, H. Zheng, B. Mao, W. Wang, C. Ye, S. Li, J. Wang, G.K.S. Wong, H. Yang, A draft sequence for the genome of the domesticated silkworm (Bombyx mori), Science (80-. ). 306 (2004) 1937-1940. doi:10.1126/science.1102210.

[23] C. Vepari, D.L. Kaplan, Silk as a biomaterial, Prog. Polym. Sci. 32 (2007) 9911007. doi:10.1016/j.progpolymsci.2007.05.013.

[24] A.R. Murphy, D.L. Kaplan, Biomedical applications of chemically-modified silk fibroin, J. Mater. Chem. 19 (2009) 6443. doi:10.1039/b905802h.

[25] N. Minoura, S.I. Aiba, M. Higuchi, Y. Gotoh, M. Tsukada, Y. Imai, Attachment and growth of fibroblast cells on silk fibroin, 208 (1995) 511-516. doi:10.1006/bbrc.1995.1368.

[26] S. Sofia, M.B. McCarthy, G. Gronowicz, D.L. Kaplan, Functionalized silk-based biomaterials for bone formation., J. Biomed. Mater. Res. 54 (2001) 139-48. http://www.ncbi.nlm.nih.gov/pubmed/11077413 (accessed June 30, 2017).

[27] G.H. Altman, F. Diaz, C. Jakuba, T. Calabro, R.L. Horan, J. Chen, H. Lu, J. Richmond, D.L. Kaplan, Silk-based biomaterials., Biomaterials. 24 (2003) 40116. http://www.ncbi.nlm.nih.gov/pubmed/12423595 (accessed June 30, 2017).

[28] R.L. Horan, K. Antle, A.L. Collette, Y. Wang, J. Huang, J.E. Moreau, V. Volloch, D.L. Kaplan, G.H. Altman, In vitro degradation of silk fibroin., Biomaterials. 26 (2005) 3385-93. doi:10.1016/j.biomaterials.2004.09.020.

[29] N.H. Chi, M.C. Yang, T.W. Chung, N.K. Chou, S.S. Wang, Cardiac repair using chitosan-hyaluronan/silk fibroin patches in a rat heart model with myocardial infarction, Carbohydr Polym. $92 \quad$ (2013) 591-597. doi:10.1016/j.carbpol.2012.09.012.

[30] N.H. Chi, M.C. Yang, T.W. Chung, J.Y. Chen, N.K. Chou, S.S. Wang, Cardiac repair achieved by bone marrow mesenchymal stem cells/silk fibroin/hyaluronic acid patches in a rat of myocardial infarction model, Biomaterials. 33 (2012) 5541-5551. doi:10.1016/j.biomaterials.2012.04.030. 
[31] M.C. Yang, N.H. Chi, N.K. Chou, Y.Y. Huang, T.W. Chung, Y.L. Chang, H.C. Liu, M.J. Shieh, S.S. Wang, The influence of rat mesenchymal stem cell CD44 surface markers on cell growth, fibronectin expression, and cardiomyogenic differentiation on silk fibroin - Hyaluronic acid cardiac patches, Biomaterials. 31 (2010) 854-862. doi:10.1016/j.biomaterials.2009.09.096.

[32] J. Zhou, B. Zhang, X. Liu, L. Shi, J. Zhu, D. Wei, J. Zhong, G. Sun, D. He, Facile method to prepare silk fibroin/hyaluronic acid films for vascular endothelial growth factor release, Carbohydr Polym. 143 (2016) 301-309. doi:10.1016/j.carbpol.2016.01.023.

[33] S. Yan, M. Li, Q. Zhang, J. Wang, Blend films based on silk fibroin/hyaluronic acid, Fibers Polym. 14 (2013) 188-194. doi:10.1007/s12221-013-0188-2.

[34] C. Foss, E. Merzari, C. Migliaresi, A. Motta, Silk fibroin/hyaluronic acid 3D matrices for cartilage tissue engineering, Biomacromolecules. 14 (2013) 38-47. doi:10.1021/bm301174x.

[35] J. Jaipaew, P. Wangkulangkul, J. Meesane, P. Raungrut, P. Puttawibul, Mimicked cartilage scaffolds of silk fibroin/hyaluronic acid with stem cells for osteoarthritis surgery: Morphological, mechanical, and physical clues, Mater Sci Eng C Mater Biol Appl. 64 (2016) 173-182. doi:10.1016/j.msec.2016.03.063.

[36] Z. Fan, F. Zhang, T. Liu, B.Q. Zuo, Effect of hyaluronan molecular weight on structure and biocompatibility of silk fibroin/hyaluronan scaffolds, Int J Biol Macromol. 65 (2014) 516-523. doi:10.1016/j.ijbiomac.2014.01.058.

[37] T.W. Chung, Y.L. Chang, Silk fibroin/chitosan-hyaluronic acid versus silk fibroin scaffolds for tissue engineering: promoting cell proliferations in vitro, J Mater Sci Mater Med. 21 (2010) 1343-1351. doi:10.1007/s10856-009-3876-0.

[38] M. Garcia-Fuentes, A.J. Meinel, M. Hilbe, L. Meinel, H.P. Merkle, Silk fibroin/hyaluronan scaffolds for human mesenchymal stem cell culture in tissue engineering, Biomaterials. $\quad 30 \quad$ (2009) 5068-5076. doi:10.1016/j.biomaterials.2009.06.008.

[39] N.R. Raia, B.P. Partlow, M. McGill, E.P. Kimmerling, C.E. Ghezzi, D.L. Kaplan, Enzymatically crosslinked silk-hyaluronic acid hydrogels, Biomaterials. 131 (2017) 58-67. doi:10.1016/j.biomaterials.2017.03.046.

[40] S. Yan, Q. Zhang, J. Wang, Y. Liu, S. Lu, M. Li, D.L. Kaplan, Silk fibroin/chondroitin sulfate/hyaluronic acid ternary scaffolds for dermal tissue reconstruction, Acta Biomater. 9 (2013) 6771-6782. 
doi:10.1016/j.actbio.2013.02.016.

[41] M. Garcia-Fuentes, E. Giger, L. Meinel, H.P. Merkle, The effect of hyaluronic acid on silk fibroin conformation, Biomaterials. 29 (2008) 633-642. doi:10.1016/j.biomaterials.2007.10.024.

[42] X. Hu, Q. Lu, L. Sun, P. Cebe, X. Wang, X. Zhang, D.L. Kaplan, Biomaterials from Ultrasonication-Induced Silk Fibroin - Hyaluronic Acid Hydrogels, Biomacromolecules. 11 (2010) 3178-3188. doi:10.1021/bm1010504.

[43] Y.J. Ren, Z.Y. Zhou, B.F. Liu, Q.Y. Xu, F.Z. Cui, Preparation and characterization of fibroin/hyaluronic acid composite scaffold, Int J Biol Macromol. 44 (2009) 372-378. doi:10.1016/j.ijbiomac.2009.02.004.

[44] E. Pavlovic, M.A. Serban, X. Yu, N.J. Manesis, Cross-linked silk-hyaluronic acid compositions, US 2014/0315828A1, 2014. https:/patents.google.com/patent/US20140315828A1/en (accessed July 22, 2017).

[45] F.S. Brandt, A. Cazzaniga, Hyaluronic acid gel fillers in the management of facial aging, Clin. Interv. Aging. 3 (2008) 153-159.

[46] S.-F. Sun, Y.-J. Chou, C.-W. Hsu, W.-L. Chen, Hyaluronic acid as a treatment for ankle osteoarthritis., Curr. Rev. Musculoskelet. Med. 2 (2009) 78-82. doi:10.1007/s12178-009-9048-5.

[47] T. Yucel, M.L. Lovett, D.L. Kaplan, Silk-based biomaterials for sustained drug delivery, J. Control. Release. $190 \quad$ (2014) 381-397. doi:10.1016/j.jconrel.2014.05.059.

[48] C.J. Bettinger, K.M. Cyr, A. Matsumoto, R. Langer, J.T. Borenstein, D.L. Kaplan, Silk fibroin microfluidic devices, Adv. Mater. Mater. 19 (2007) 28472850. doi:10.1002/adma.200602487.Silk.

[49] J. Schindelin, I. Arganda-Carreras, E. Frise, V. Kaynig, M. Longair, T. Pietzsch, S. Preibisch, C. Rueden, S. Saalfeld, B. Schmid, J.-Y. Tinevez, D.J. White, V. Hartenstein, K. Eliceiri, P. Tomancak, A. Cardona, Fiji: an open-source platform for biological-image analysis, Nat. Methods. 9 (2012) 676-682. doi:10.1038/nmeth.2019.

[50] P. Taddei, E. Pavoni, M. Tsukada, Stability toward alkaline hydrolysis of B. mori silk fibroin grafted with methacrylamide, J. Raman Spectrosc. 47 (2016) 731739. doi:10.1002/jrs.4892.

[51] G.B. Perea, C. Solanas, N. Marí-Buyé, R. Madurga, F. Agulló-Rueda, A. 
[52] M. Hu, E.E. Sabelman, C. Tsai, J. Tan, V.R. Hentz, Improvement of Schwann Cell Attachment and Proliferation on Modified Hyaluronic Acid Strands by Polylysine, Tissue Eng. 6 (2000) 585-593. doi:10.1089/10763270050199532.

[53] Y. Jo, H. Kweon, K. Lee, S. Nam, H. Lee, J. Yeo, The Promotion of Cell Attachment and Proliferation on Silk Fibroin, 54 (2011) 166-170.

[54] G.A. Monteiro, A. V Fernandes, H.G. Sundararaghavan, D.I. Shreiber, Positively and Negatively Modulating Cell Adhesion to Type I Collagen Via Peptide Grafting, Tissue Eng. Part A. 17 (2011) 1663-1673. doi:10.1089/ten.tea.2008.0346.

[55] C. Calcagno, M.E. Lobatto, P.M. Robson, A. Millon, Mapping structural landmarks, ligand binding sites and missense mutations to the collagen IV heterotrimers predicts major functional domains, novel interactions and variation in phenotypes in inherited diseases affecting basement membranes, Hum Mutat. 28 (2016) 1304-1314. doi:10.1002/nbm.3369.Three.

[56] A.U. Ude, R.A. Eshkoor, R. Zulkifili, A.K. Ariffin, A.W. Dzuraidah, C.H. Azhari, Bombyx mori silk fibre and its composite: A review of contemporary developments, Mater. Des. $57 \quad$ (2014) 298-305. doi:10.1016/j.matdes.2013.12.052.

[57] E.D.T. Atkins, C.F. Phelps, J.K. Sheehan, The conformation of the mucopolysaccharides. Hyaluronates, Biochem. J. 128 (1972) 1255-1263. doi:10.1042/bj1281255. 
Fig. 1. A: Experimental thermograms of $\mathrm{NaOH}$-treated SF (dotted line), water-treated (solid line) and untreated SF (dashed line). Values are expressed as mean $\pm \mathrm{SD}$. The thermograms show that SF exposed to a basic medium suffers a chemical alteration. B: Experimental thermograms of HA (solid line), HA-SF (dashed line) and $\mathrm{NaOH}$-treated $\mathrm{SF}$ (dotted line). Values are expressed as mean $\pm \mathrm{SD}$. In the temperature range between $220{ }^{\circ} \mathrm{C}$ and $370{ }^{\circ} \mathrm{C}$ there is an intermediate behavior of the HA-SF thermogram, indicating the presence of SF. The theoretical and the experimental mass fraction of SF in the HA-SF material is also indicated.

Fig. 2. Storage modulus (A) and loss modulus (B) as a function of the oscillation torque for HA (circles) and HA-SF (squares). Values are expressed as mean \pm SD. Presence of SF increases both the storage and the loss moduli.

Fig. 3. A-D: Confocal microscopy images corresponding to rSCs cultured on HA for 1 (A) and 5 days (B) and on HA-SF for 1 (C) and 5 days (D). The cytoskeleton is shown in green and the nuclei are shown in blue. Scale bar $=30 \mu \mathrm{m}$. E: Total area of rSCs cytoskeleta after 1 and 5 days of culture on HA and HA-SF. Cells cultured on HA-SF films presented a larger cytoskeleton area when compared to HA films, indicating a greater cell adhesion thanks to the presence of SF.

Fig. 4. FESEM images of axial cuts and cross sections of HA (A, B and $\left.B^{\prime}\right)$ and HA-SF $\left(\mathrm{C}, \mathrm{D}\right.$ and $\left.\mathrm{D}^{\prime}\right)$ conduits. $\mathrm{B}^{\prime}$ and $\mathrm{D}^{\prime}$ show a detail of the internal surface of the conduit. Scale bar $=500 \mu \mathrm{m}(\mathrm{A}, \mathrm{B}, \mathrm{C}$ and $\mathrm{D})$ and $100 \mu \mathrm{m}\left(\mathrm{B}^{\prime}\right.$ and $\left.\mathrm{D}^{\prime}\right)$. Matrix porosity is produced by the lyophilization process. The inner channel surface is less porous, due to the hydrophobic nature of the channel template. Arrows in D' indicate the presence of elongated structures on the surface of HA-SF conduits that may correspond to SF fibers.

Fig. 5. Confocal microscopy images corresponding to rSCs cultured inside HA tubes for 1 (A), 5 (B) and 10 days (C), and inside HA-SF tubes for 1 (D), 5 (E) and 10 days (F). The cytoskeleton is shown in green and the nuclei are shown in blue. Scale bars $=100$ $\mu \mathrm{m}$. The presence of SF seems to accelerate the formation of the cell sheath, which is completely developed in the HA-SF conduits at day 5 (arrows in E showing a fold of this structure), while it took 10 days to fully develop in HA tubes (arrows in C).

Fig. 6. MTS proliferation assays on rSCs cultures inside HA and HA-SF conduits at different culture times (1, 5 and 10 days). The presence of SF increases cell proliferation, explaining the earlier formation of the cell sheath in the HA-SF conduits.

Fig. 7. HA and HA-SF scaffolds were implanted subcutaneously by means of two independent skin incision of approximately $1 \mathrm{~cm}$ on the back of each mouse. The skin sections containing the scaffolds were photographed at 1 (A and B for HA and HA-SF scaffolds, respectively), 4 (C and D for HA and HA-SF scaffolds, respectively) and 8 (E and $\mathrm{F}$ for HA and HA-SF scaffolds, respectively) weeks after their implantation. Healthy tissue can be observed surrounding the scaffolds since week 1. A high 
capillarity (white arrows) that is near to or in direct contact with the scaffolds is also visible since the first week. Scale bars $=2 \mathrm{~mm}$.

Fig. 8. Cross sections of representative HA scaffolds stained with H\&E and Masson's trichrome. One week after the implantation of the scaffold, an acute moderate foreign body reaction can be observed, with a large infiltration of granulocyte populations (A and B for H\&E staining, C and D for Masson's trichrome staining). A fibrous capsule surrounding the scaffold is clearly visible which is a typical reaction after the implantation of a biomaterial. After 4 weeks, a reduction of the immune response can be observed, corresponding to a mild foreign body reaction ( $E$ and $F$ for $H \& E$ staining, $G$ and $\mathrm{H}$ for Masson's trichrome staining). The population of granulocytes has been reduced and there is a greater presence of macrophages. After 8 weeks, the scaffold has been completely resorbed and assimilated into the native tissue (I and $\mathrm{J}$ for $\mathrm{H} \& \mathrm{E}$ staining, K and L for Masson's trichrome staining). The fibrous capsule has disappeared and there are no granulocytes present. Now the cells present are mainly macrophages and active fibroblasts. Also, an increase of collagen structures inside the scaffold can be observed thanks to the fact that active fibroblasts have managed to invade the biomaterial. Many cross-sections of blood vessels are also visible after 8 weeks. Scale bar $=2 \mathrm{~mm}(\mathrm{~A}, \mathrm{C}, \mathrm{E}, \mathrm{G}, \mathrm{I}$ and $\mathrm{K})$ and $500 \mu \mathrm{m}(\mathrm{B}, \mathrm{D}, \mathrm{F}, \mathrm{H}, \mathrm{J}$ and $\mathrm{L})$.

Fig. 9. Cross sections of representative HA-SF scaffolds stained with H\&E and Masson's trichrome. One week after the implantation of the scaffold a fibrous capsule surrounding the scaffold is clearly visible, corresponding to an acute moderate foreign body reaction (A and B for H\&E staining, C and D for Masson's trichrome staining). An infiltration of granulocyte populations is also observed. After 4 weeks, a clear reduction of the immune response can be observed, since there is a clear presence of blood vessels and collagen networks inside the scaffold and the population of granulocytes has been reduced ( $\mathrm{E}$ and $\mathrm{F}$ for H\&E staining, $\mathrm{G}$ and $\mathrm{H}$ for Masson's trichrome staining). After 8 weeks, the fibrous capsule has disappeared and there are no granulocytes present, so the scaffold has been completely resorbed and assimilated into the native tissue ( $\mathrm{I}$ and $\mathrm{J}$ for H\&E staining, $\mathrm{K}$ and L for Masson's trichrome staining). Now the cells present are mainly macrophages and active fibroblasts. Many collagen structures and cross-sections of blood vessels can be observed. Scale bar $=2 \mathrm{~mm}$ (A, C, $\mathrm{E}, \mathrm{G}, \mathrm{I}$ and $\mathrm{K})$ and $500 \mu \mathrm{m}(\mathrm{B}, \mathrm{D}, \mathrm{F}, \mathrm{H}, \mathrm{J}$ and L).

Fig. 10. Details of cross sections of representative HA and HA-SF scaffolds after 8 weeks of implantation. A and B: HA scaffold stained with Masson's trichrome. C and D: HA-SF scaffold stained with Masson's trichrome. In these images both the collagen deposition and the vascularization of HA and HA-SF scaffolds after 8 weeks of implantation are observable. Multiple collagen fibres (blue arrows) surrounded by active fibroblasts (green arrows) and cross-sections of blood vessels (red arrows) within the scaffold confirm the deposition of new extracellular matrix and the process of angiogenesis, respectively. The scaffold walls are indicated by black arrows. Scale bar = $200 \mu \mathrm{m}(\mathrm{A}, \mathrm{C})$ and $100 \mu \mathrm{m}(\mathrm{B}, \mathrm{D})$. 

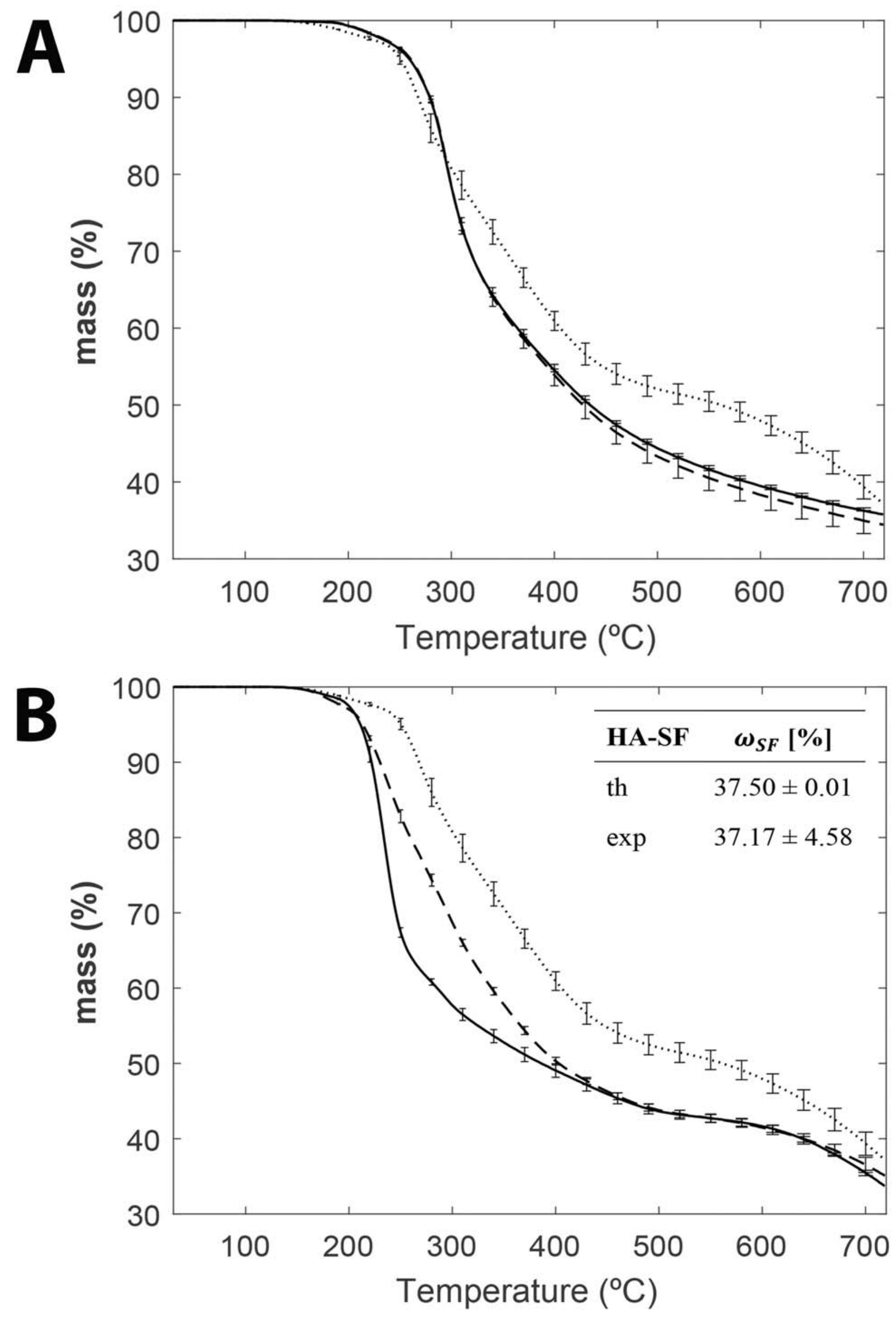

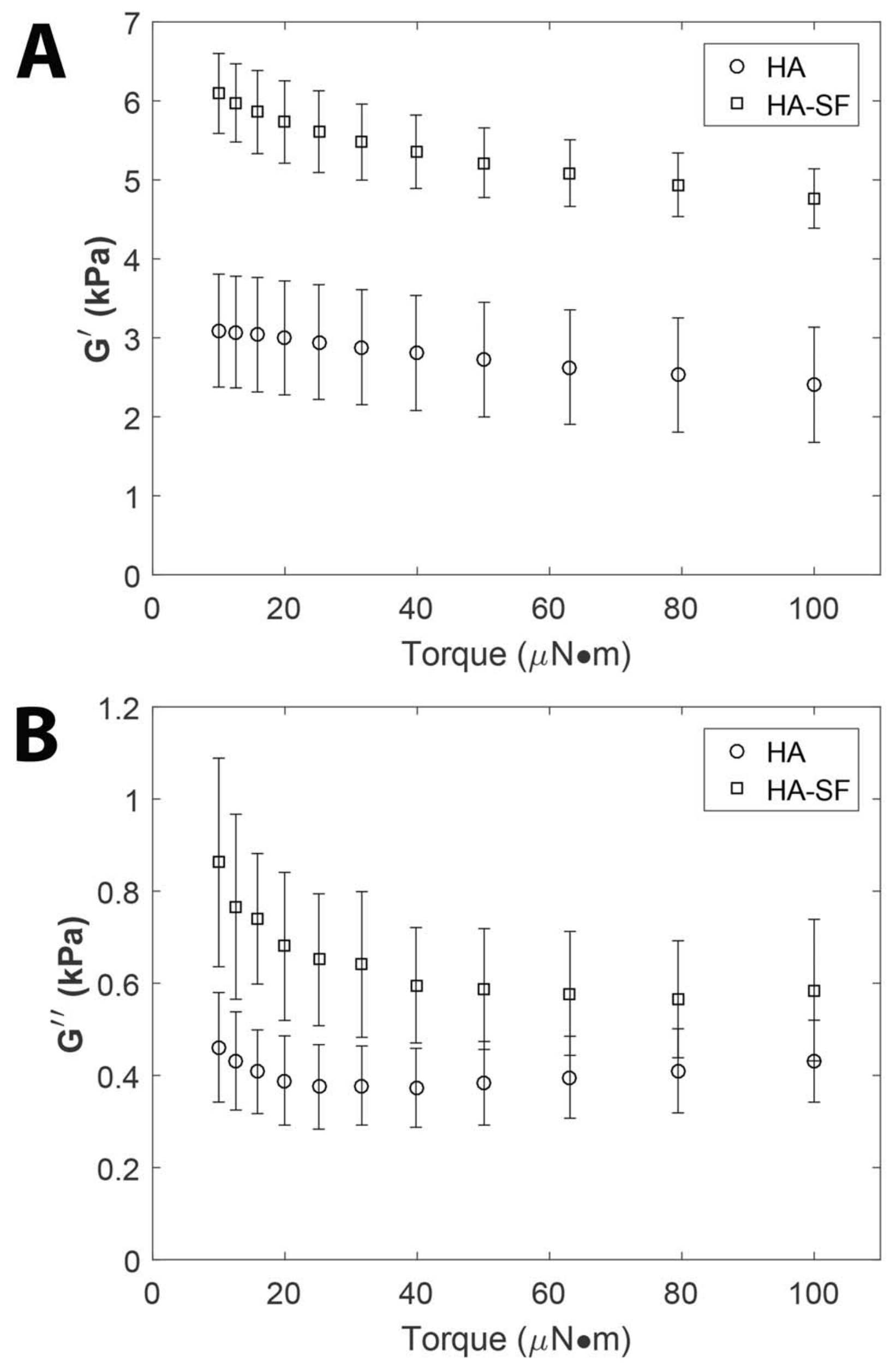

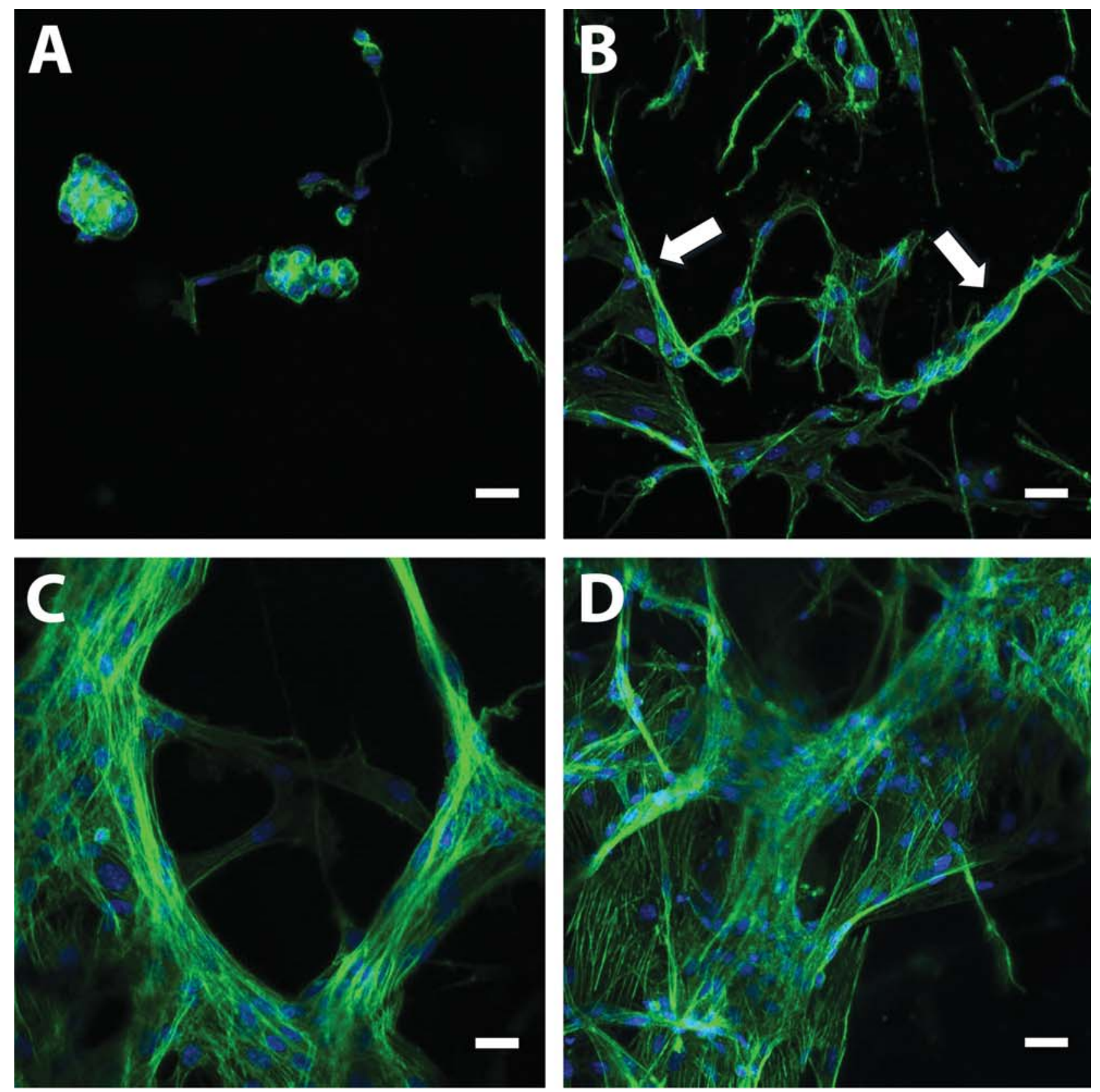

\section{E}

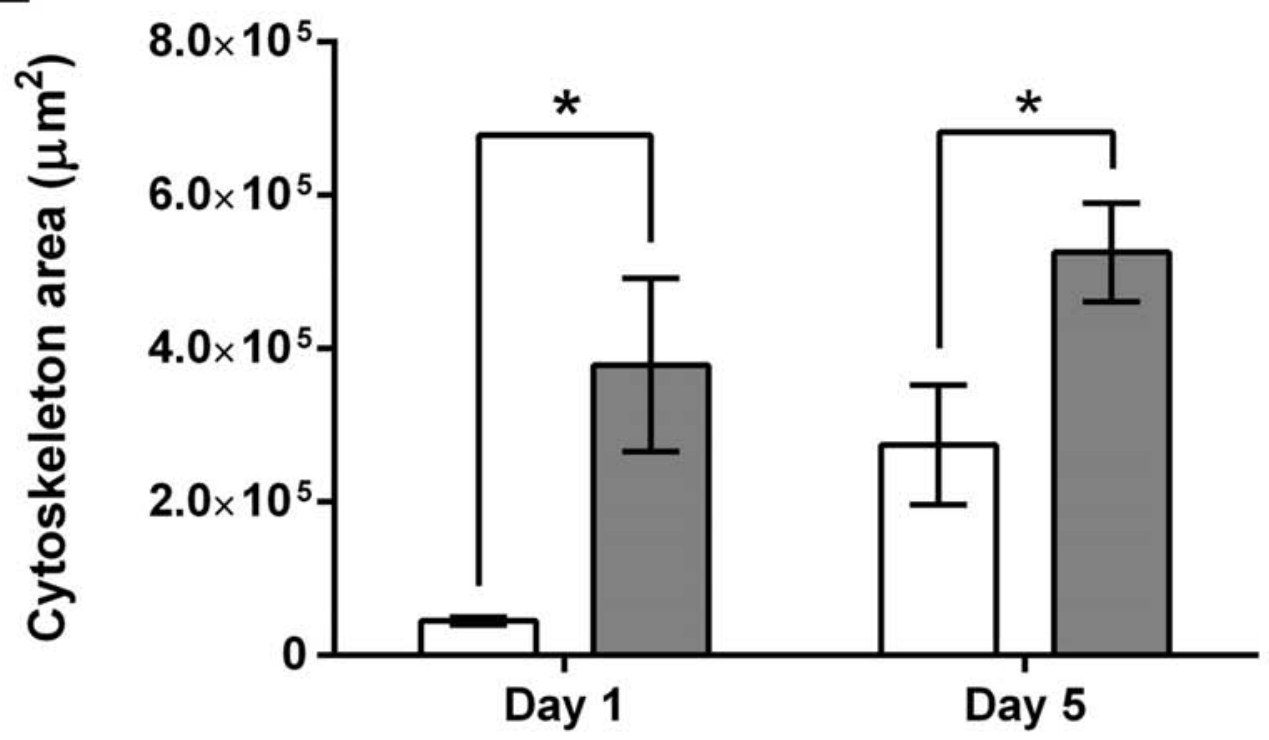

$\square \mathrm{HA}$

$\square$ HA-SF 

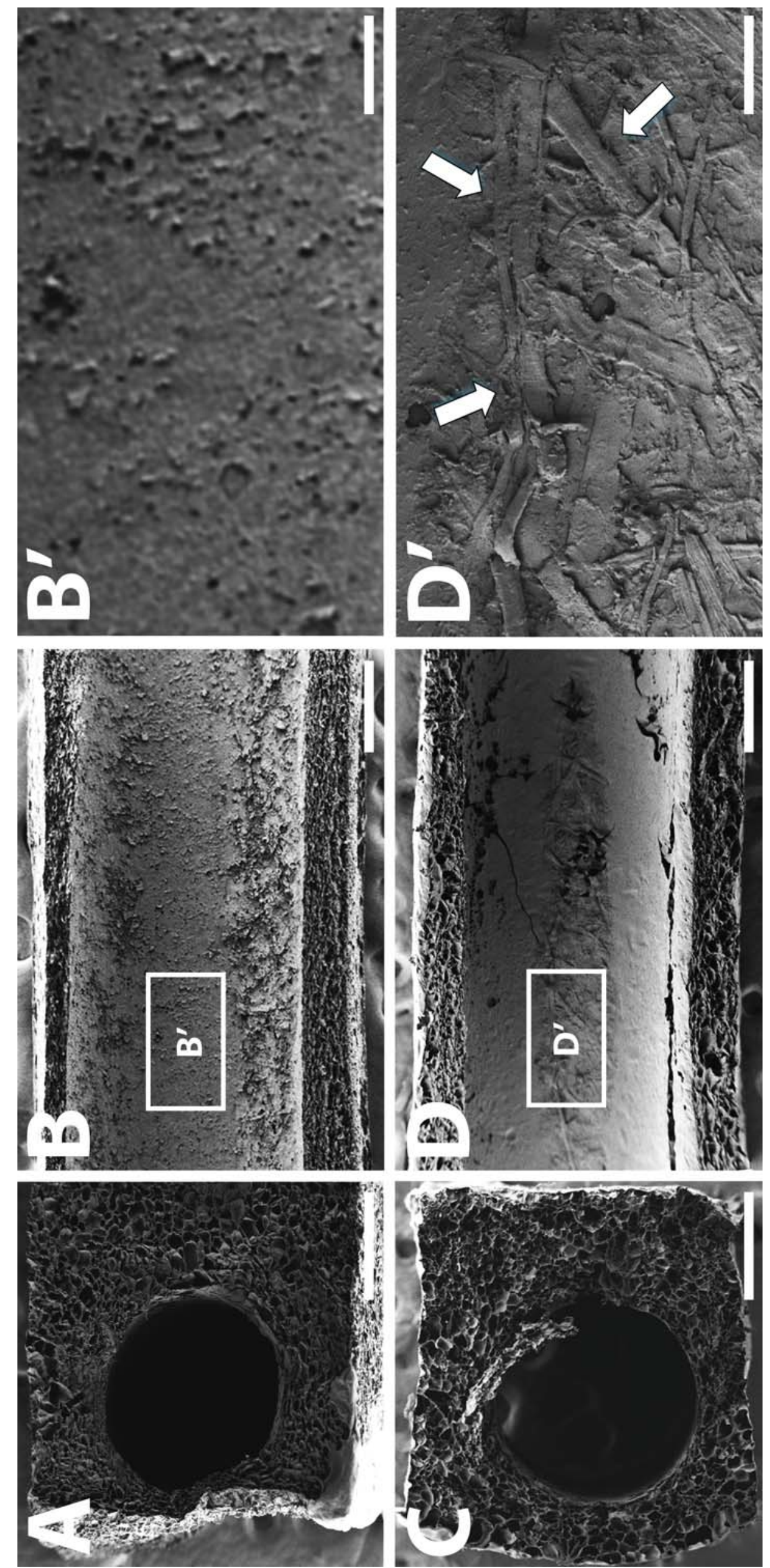

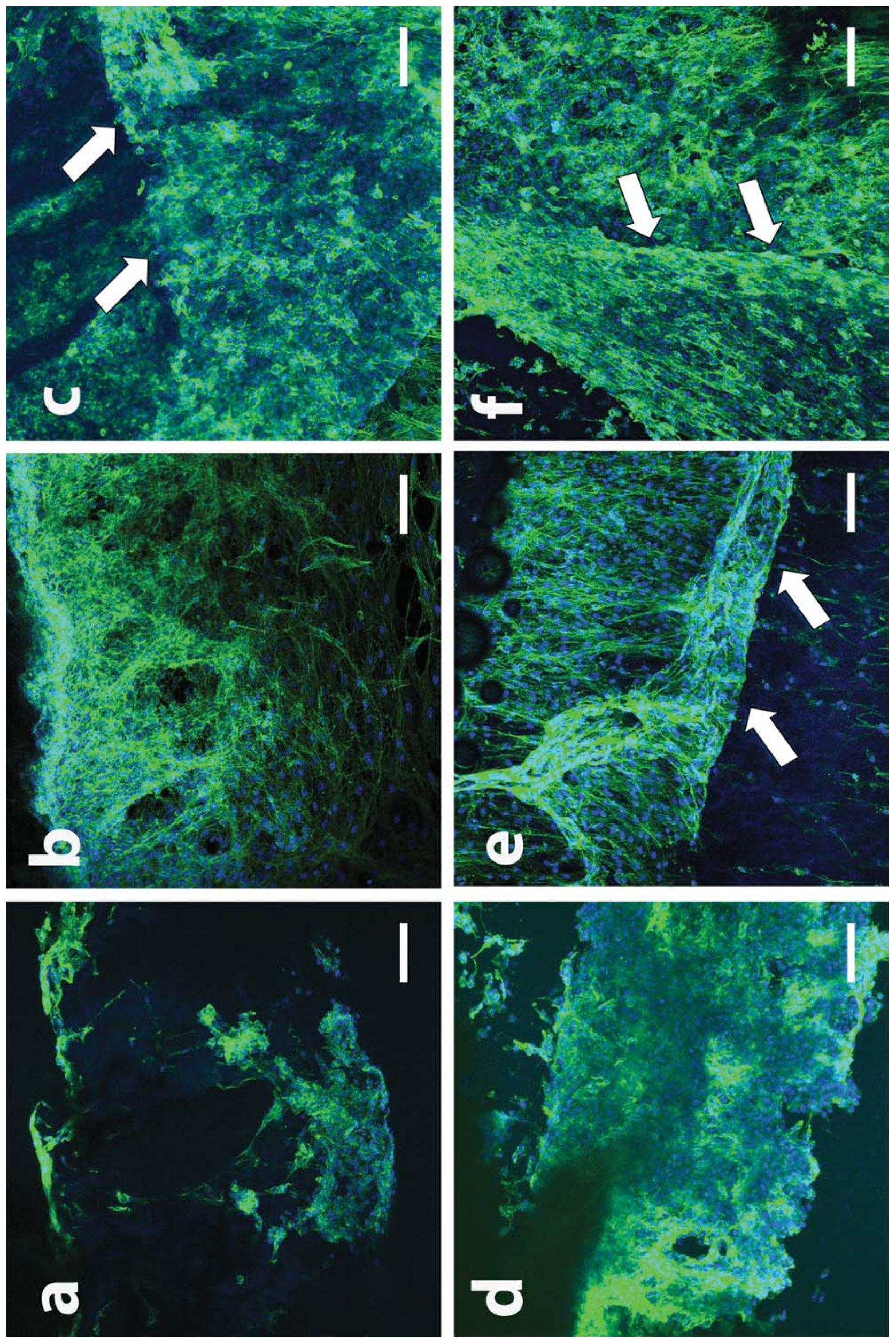
$\frac{1}{\omega}$
交
至
00
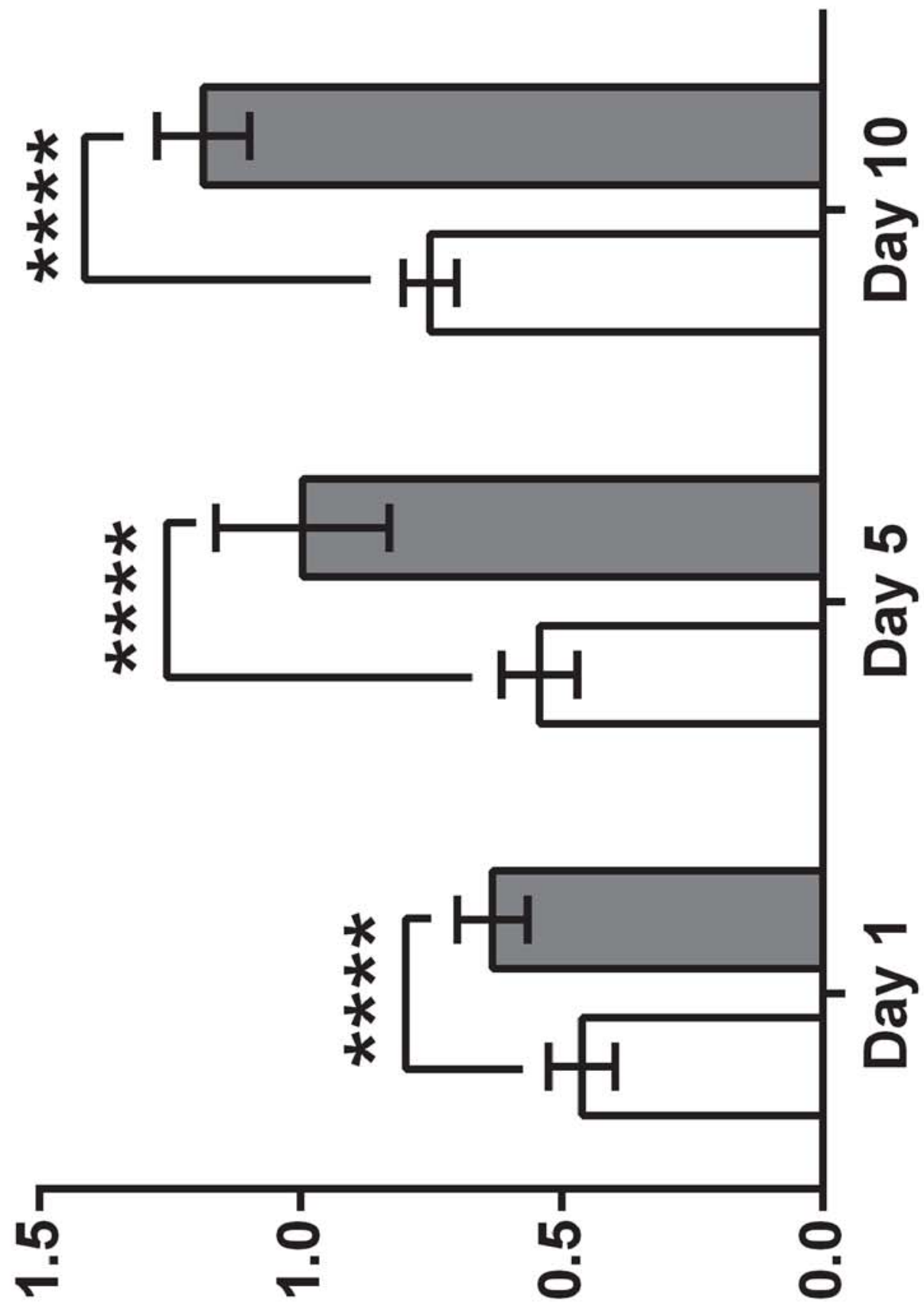

('n'e) әourequosq 

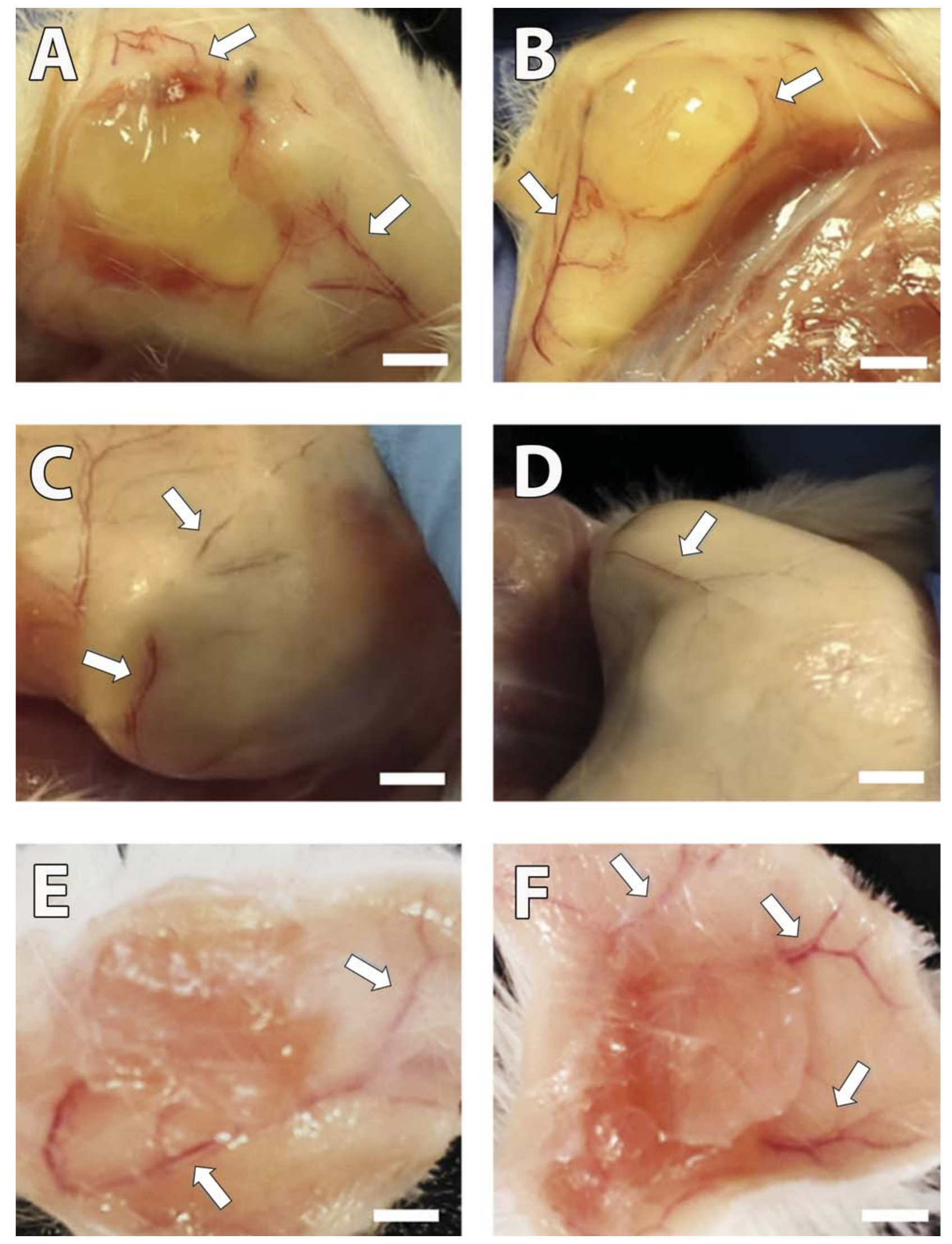

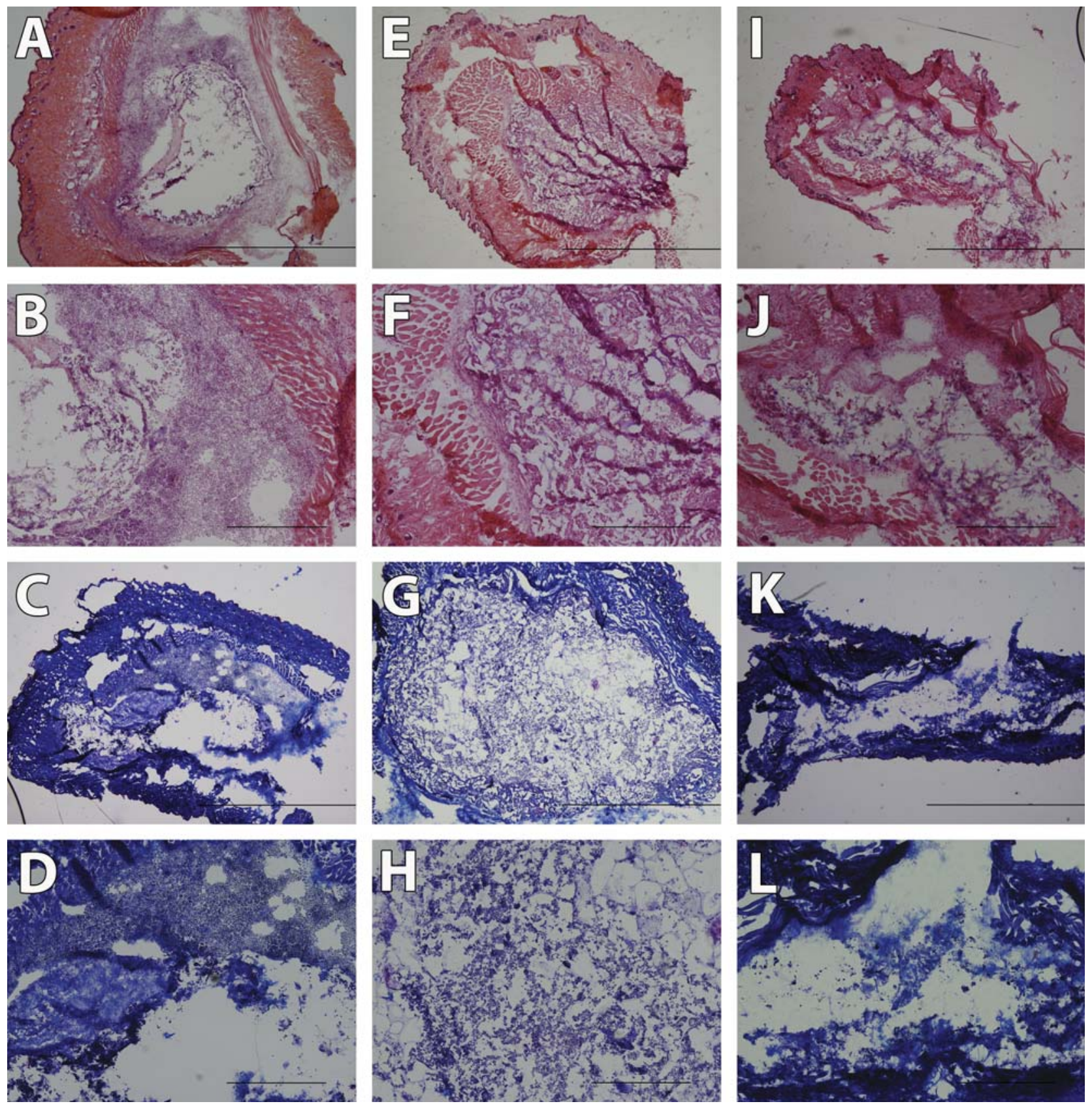

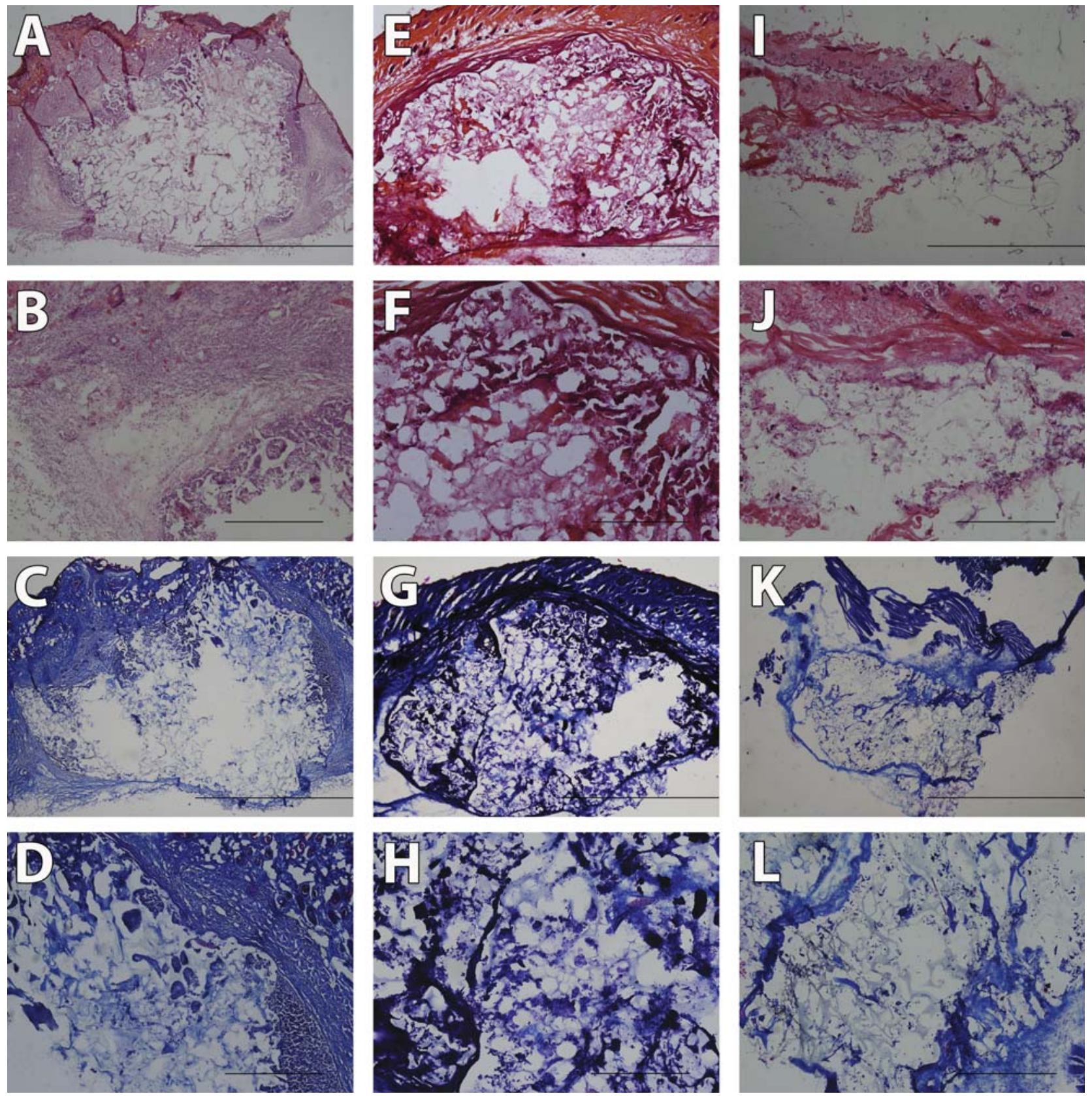

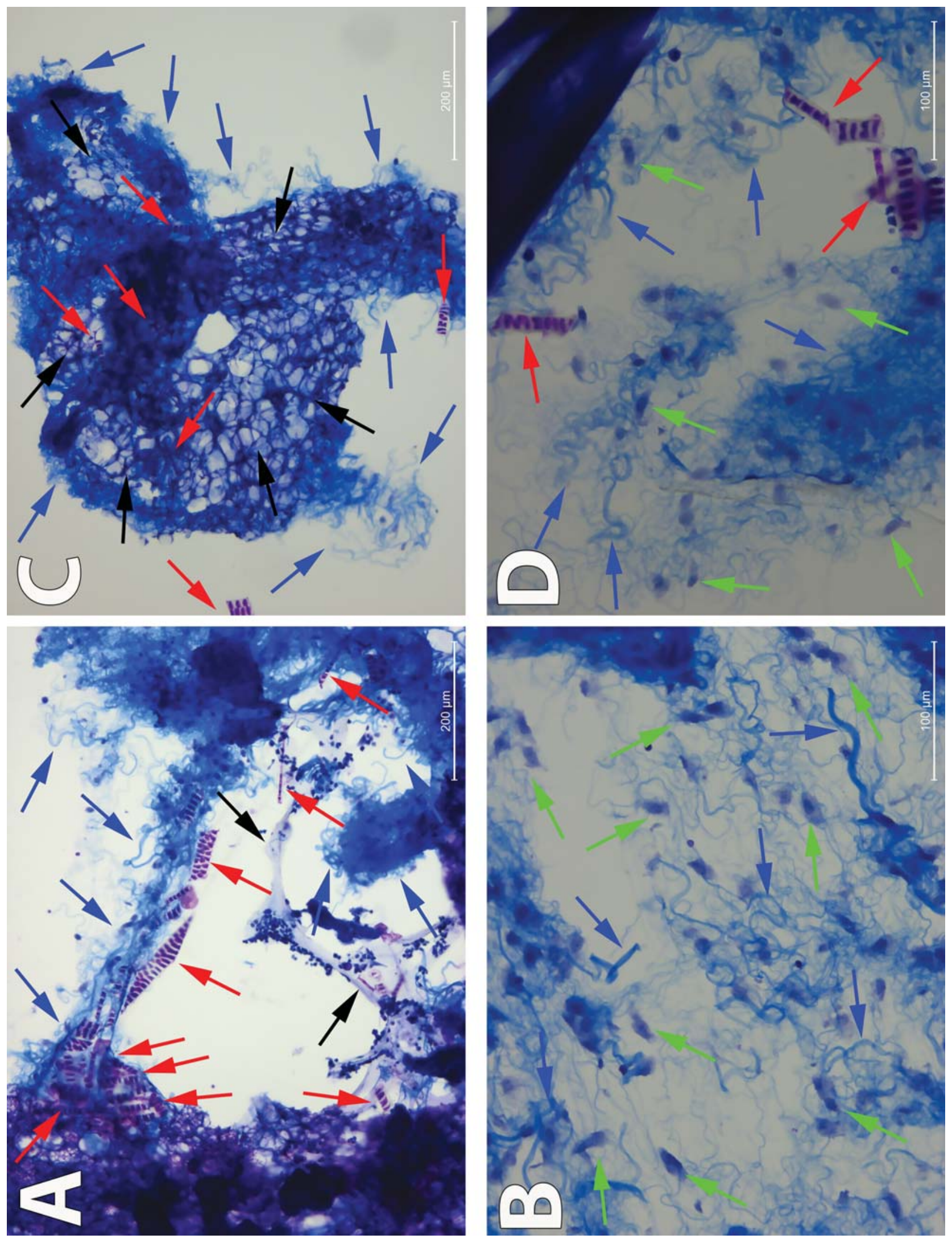
The corresponding authors confirm that all authors have seen and approved the manuscript to be considered for publication in International Journal of Biological Macromolecules.

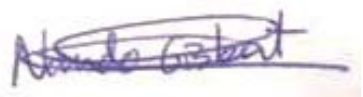

\section{Fernando Gisbert Roca}

Center for Biomaterials and Tissue Engineering, Universitat Politècnica de València, Cno. de Vera s/n, 46022 Valencia, Spain

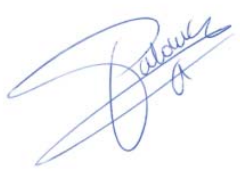

\section{Paloma Lozano Picazo}

CIBER-BBN, Biomedical Research Networking Center in Bioengineering Biomaterials and Nanomedicine, Spain. Centro de Tecnología Biomédica.

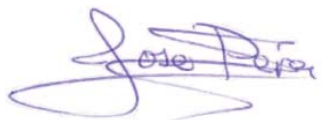

\section{Jose Pérez Rigueiro}

CIBER-BBN, Biomedical Research Networking Center in Bioengineering Biomaterials and Nanomedicine, Spain. Centro de Tecnología Biomédica. Universidad Politécnica de Madrid. Departamento de Ciencia de Materiales. ETSI Caminos, Canales y Puertos., Universidad Politécnica de Madrid.

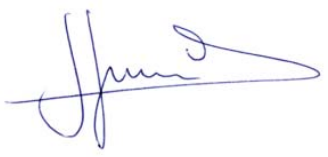

\section{Gustavo Victor Guinea}

CIBER-BBN, Biomedical Research Networking Center in Bioengineering Biomaterials and Nanomedicine, Spain. Centro de Tecnología Biomédica. Universidad Politécnica de Madrid. Departamento de Ciencia de Materiales. ETSI Caminos, Canales y Puertos., Universidad Politécnica de Madrid.

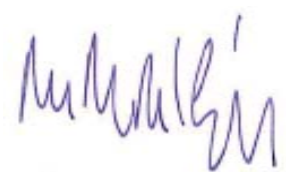

\section{Manuel Monleón Pradas}

Center for Biomaterials and Tissue Engineering, Universitat Politècnica de València, Cno. de Vera s/n, 46022 Valencia, Spain

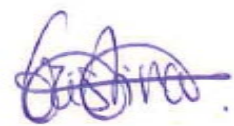

\section{Cristina Martínez-Ramos}

Center for Biomaterials and Tissue Engineering, Universitat Politècnica de València, Cno. de Vera s/n, 46022 Valencia, Spain 